\title{
Characterization Procedure for Stand-Alone Merging Units Based on Hardware-in-the-Loop Technology
}

\author{
Alessandro Mingotti *(D), Federica Costa $(D)$, Lorenzo Peretto (D) and Roberto Tinarelli $(\mathbb{D}$ \\ Department of Electrical, Electronic and Information Engineering, Guglielmo Marconi, Alma Mater Studiorum, \\ University of Bologna, Viale del Risorgimento 2, 40136 Bologna, Italy; federica.costa13@unibo.it (F.C.); \\ lorenzo.peretto@unibo.it (L.P.); roberto.tinarelli3@unibo.it (R.T.) \\ * Correspondence: alessandro.mingotti2@unibo.it
}

Citation: Mingotti, A.; Costa, F.;

Peretto, L.; Tinarelli, R.

Characterization Procedure for Stand-Alone Merging Units Based on Hardware-in-the-Loop Technology. Energies 2021, 14, 1993. https:// doi.org/10.3390/en14071993

Academic Editor: Paolo Attilio Pegoraro

Received: 1 March 2021

Accepted: 30 March 2021

Published: 4 April 2021

Publisher's Note: MDPI stays neutral with regard to jurisdictional claims in published maps and institutional affiliations.

Copyright: (c) 2021 by the authors. Licensee MDPI, Basel, Switzerland. This article is an open access article distributed under the terms and conditions of the Creative Commons Attribution (CC BY) license (https:// creativecommons.org/licenses/by/ $4.0 /)$.
Abstract: The digitalization of a medium voltage network requires huge efforts from distributed system operators and electric utilities. The main reason is attributed to the costs associated with the replacement or introduction of new intelligent electronic devices capable of collecting and digitalizing current and voltage measurements. To this purpose, this paper introduces a new idea of a stand-alone merging unit (SAMU), which features real-time and hardware-in-the-loop technology, completed with accurate voltage and current sensors. Furthermore, the characterization procedure that allows an evaluation of the metrological performance of a complex device, such as a SAMU, is fully described. From the results, it is highlighted that (i) the developed SAMU is capable of performing highly accurate voltage, current, and power measurements; (ii) the characterization procedure is simple and exploitable for all kinds of SAMUs and other synchronized measurement devices.

Keywords: merging unit; stand-alone merging unit; characterization; hardware-in-the-loop; realtime; OPAL; accuracy; instrument transformer

\section{Introduction}

A significant step towards the transition of a power network into a Smart Grid consists of the digitalization of the network and, in particular, the substations. If the digitalization of primary substations may be an "easy" task (e.g., Italy has fewer than one thousand primary substations), digitalizing the secondary or distribution substations is not as easy (between one and two orders of magnitude more so than the primary ones). Among the obstacles for Distribution System Operators (DSOs) reaching a full digitalization of medium and low voltage (MV and LV, respectively) is part of the network, such as [1]: (i) the MV/LV network topology. In fact, MV and LV networks have a meshed structure with a huge number of nodes. Furthermore, typically, the distribution part of the network extends itself to rural areas, or is really complicated in its attempts to reach such areas (mountains, hills, etc.), to provide energy to all customers. Another obstacle is (ii): economic availability. This second reason is connected to the previous one. In other words, a DSOs investments for the grid are reasonably distributed among all nodes of the network, resulting in a low ratio of funds per node (compared to a transmission network).

Therefore, what can be perceived from the network is that the transmission and the distribution grids are being digitalized at different growing rates.

Focusing on the secondary substations, which is the aim of this paper, they mainly consist of a few fundamental electric apparatuses: circuit-breakers and protections [2-4], cable accessories and terminations [5-8], power transformers [9,10], and instrument transformers. In addition, communication infrastructure (copper or fiber optic-based) completes the substation. Such infrastructure must carry the information gathered from the measurement devices to the control rooms in order for the operators to make the correct management choices. 
Considering that the measurements performed inside the secondary substations are used for triggering protective devices, and are used as a base for important controlling decisions, instrument transformers (ITs) play a fundamental role. To this purpose, researchers studied, and currently are studying, every peculiar aspect of their operation and design technologies. In [11-13], it has been approached and developed for the modelling of typical ITs and of the new generation of Low-Power Instrument Transformers (LPITs). Frequency characterization procedures for ITs have been proposed in [14,15]; whereas in $[16,17]$, characterization has been addressed with specific distorted testing waveforms to be applied to ITs and LPITs. Finally, another fundamental topic to be investigated is the influence of typical external or internal factors on the transformers' accuracy. Therefore, in [18-22], the influence of geometry, burden, magnetic field, and temperature, on both ITs and LPITs, is assessed and described.

Furthermore, Technical Committees (TCs) and standard organizations are making a huge effort to help manufacturers and researchers in publishing up to date documents that cover the peculiarities of ITs and their operations.

This huge effort resulted in a new series of standards completely dedicated to ITs: the IEC 61869. Document IEC 61869-1 and -6 [23,24] provide general definitions and guidelines for the legacy inductive and the new generation of LPITs, respectively (also referred to as nonconventional instrument transformers). Inside the IEC 61869 series, documents -2 and $-3[25,26]$ deal with inductive current and voltage transformers (CTs and VTs), respectively; while documents -10 and $-11[27,28]$ describe the Low-Power Current Transformers (LPCTs) and the Low-Power Voltage Transformers (LPVTs), respectively.

Even if LPITs brought novelties and smartness to the secondary substations, the idea of a fully digitized substation involves a further step. It includes the digitalization and synchronization of the acquired measurements according to specific protocols. In this process, a new device that is being installed and studied more and more, including this paper, is the so-called Merging Unit (MU), or Stand-Alone Merging Unit (SAMU). As it is better described in Section 2, including the difference between an MU and a SAMU, it allows a merging of the secondary outputs of the ITs and LPITs. In addition, it associates a timestamp with each set of measurements to guarantee global synchronization that may be used to assess measurements coming from different MUs.

MUs, as with other devices, must fulfill some standardized requirements, including accuracy limits and communication protocols. Therefore, researchers are trying to find new ways of testing Mus by considering their communication and synchronization aspects. For example, the early-stage phase of MU development allows researchers to propose their own MU solutions. In [29,30], a basic design of an MU, based on the standards, is presented. MUs based on dual CPU or on ARM technology are described in [31] and [32,33], respectively. A new concept of MU, combined with the functionalities of a Phasor Measurement Unit (PMU), is introduced in [34].

Turning to the performance assessment of an MU (or SAMU), two studies focused on the calibration equipment needed for accuracy tests are discussed in [35,36]. In [37], instead, the calibration process is completed without the need for a synchronization system. An accuracy evaluation focused on the phase angle evaluation is tackled in [38]. In [39] and [40], instead, accuracy analysis is based on specific testing signals and on triangular fuzzy numbers, respectively.

In light of the above, this article aims to introduce a different perspective on the MUs scenario. In particular, the concept of an MU is combined with hardware-in-the-loop (HIL) and real-time technologies that are becoming, day by day, more popular, and are being adopted by several research institutes and electrical utilities. Therefore, this paper presents a SAMU based on real-time HIL technology, as well as the characterization procedure that allows us to assess its performance from a metrological perspective. The added value of combining such significant technologies, hence, of this work, is that the features of a SAMU can be implemented and tested in an HIL environment, allowing us to simulate a complete digital substation inside the laboratory. What is presented in the following allows 
us to exploit existing technologies of a laboratory environment for the development of new equipment with additional functionalities.

The remainder of the work is structured as follows. Section 2 provides the basic concepts and the standard vision of the SAMU, including communication and accuracy aspects. The measurement setup that recreates a SAMU is described in Section 3. Section 4 details the characterization procedure of the entire SAMU and the single components, which includes a better understanding of the influence of each component. The results are listed and discussed in Section 5, where the accuracy of the proposed SAMU is confirmed. Finally, Section 6 summarizes the main achievements of the work by drawing some conclusions.

\section{Stand-Alone Merging Unit}

This Section is completely dedicated to the SAMU. Considering the available standards, the aim is to clarify the definitions and working principles related to a SAMU. Therefore, Section 2.1 introduces the definitions of a SAMU, starting from the logical device concept. In Section 2.2 a summary of the working principle of a SAMU is provided. In Section 2.3, a brief summary of the fundamental communication aspects is provided. The accuracy and associated tests are presented in Section 2.4. Finally, Section 2.5 contains the main characteristics of the SAMU that has been designed in this work and is fully described in Section 3.

\subsection{Definition}

To understand what a SAMU is, one may start from IEC 61869-6 [24], where a SAMU is defined as a "merging unit with standardized inputs (analogue or digital)". Therefore, the main difference between an MU and a SAMU is the fact of having specific input values, which cannot be generic. The second step is the definition of an MU. To this purpose, two standards have to be considered:

- IEC 61869-6 [24] defines an MU as "physical device (IED according to IEC 61850-2) in which a logical device merging unit is implemented"; and a logical device merging unit is a "logical device (in the meaning of IEC 61850-7-4) to do the time-coherent combination of logical nodes current transformer (TCTR) and/or logical nodes voltage transformer (TVTR) for building a standard digital output". Consequently, to complete the MU definition, IEC 61850-2 [41] has to be used, as it contains the definition of a logical device: an "entity that represents a set of typical automation, protection or other functions".

- IEC 61850-2 [41] provides two definitions of an MU: one is a "physical unit performing the time coherent combination of the current and/or voltage data coming from sensors"; while the second is an "interface unit that accepts multiple analogue CT/VT and produces multiple time synchronized IEC 61850-9-2 compliant frames to provide data communication via the logical interface 4 ".

As can be noted, there is no complete agreement between the potential definitions. Instead, slight differences can be found between [24] and [41]. In particular, following [24], the MU definition is quite long, and it relies on several sub-definitions. Starting from [41], instead, it is easy to reach a clear definition, even if there are two versions of the same definition. Finally, in 02/21, the new IEC 61869-13 [42] was published, completely dedicated to SAMU. However, no definition of it is provided; hence, it can be assumed that what defined in [24] is still valid and confirmed.

\subsection{Working Principle}

With the help of Figure 1, the structure and working principle of a SAMU can be described. It consists of a unit that collects the output signals of instrument transformers, both legacy ITs and LPITs. In detail, Ref. [42] defines rated inputs for legacy inductive transformers as $1 \mathrm{~A}$ and $5 \mathrm{~A}$, and values between $100 \mathrm{~V}$ and $200 \mathrm{~V}$ for CTs and VTs, respectively. For the LPITs, instead, the standard defines $22.5 \mathrm{mV}, 150 \mathrm{mV}$, and $225 \mathrm{mV}$ 
for the current one, and $3.25 \mathrm{~V}$ for the voltage one. Furthermore, a SAMU is capable of colleting digital inputs from the ITs, in addition to the typical analogue ones.

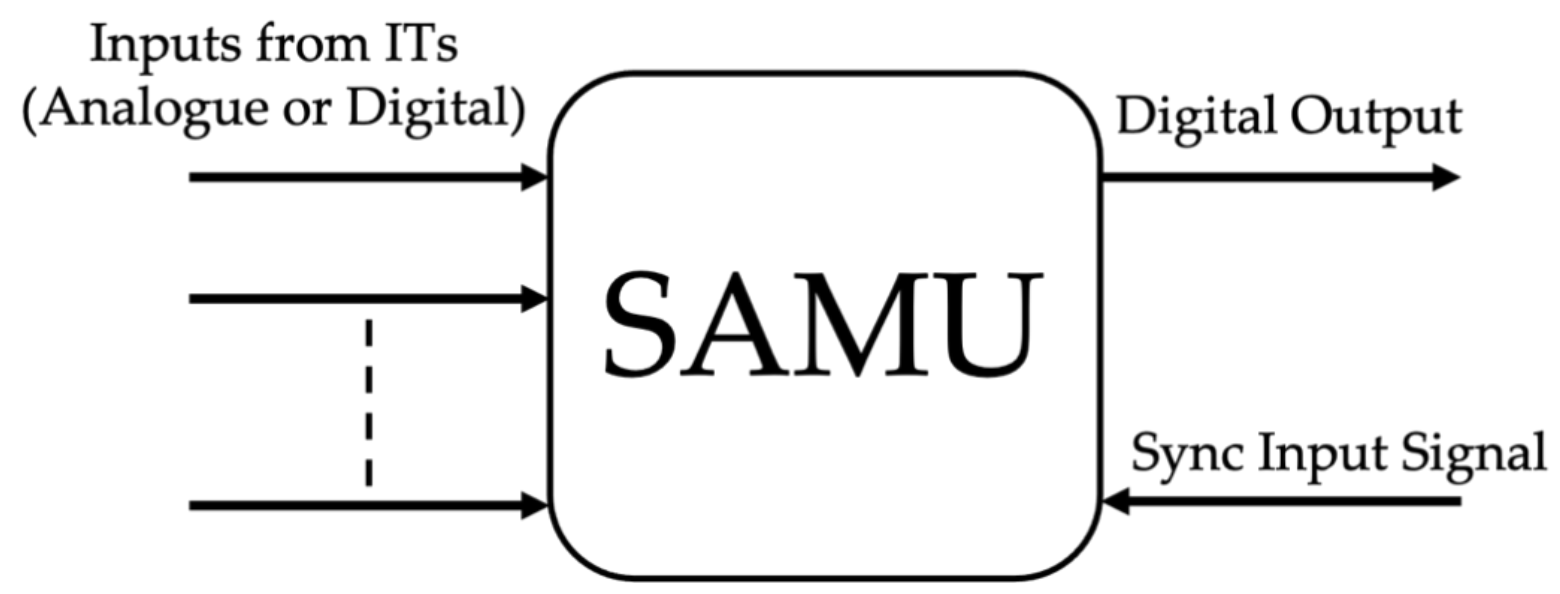

Figure 1. General structure of a stand-alone merging unit (SAMU), according to [42].

Turning to the output of the SAMU, it consists of a digital signal, in accordance with what is defined in IEC 61850-9-2 [43] (more details on communication are provided in the next, Section 2.3).

Finally, what completes a SAMU and confers it an added value, is the synchronization input signal. It allows synchronization of the data sampling of the ITs outputs with an external signal that could be shared among several SAMUs. An example of this external synchronization signal is the well-known pulse per second (PPS). Therefore, at each rising front of the PPS, the SAMU starts data collection and aligns its internal clock with the PPS.

The drawback of the working principle of a SAMU manifests if the synchronization is lost, resulting in a downgrade of a SAMU to a simple asynchronous data collector.

\subsection{Communication}

The communication requirements of a SAMU are standardized by the IEC 61850 series and the IEC 61869-9 [44]. In particular, the latter standard is an application of the IEC 61850 specifically developed for ITs. Furthermore, it includes details on the sample rates, time synchronization, and control blocks related to SAMUs.

Starting at the sample rates, Ref. [44] defines eight combinations of sample rates and publishing rates, ranging from $4800 \mathrm{Sa} / \mathrm{s}$ to $96,000 \mathrm{Sa} / \mathrm{s}$. Among those rates, some are specific for $50 \mathrm{~Hz}$ systems and others for the $60 \mathrm{~Hz}$ ones.

Turning to synchronization, a SAMU uses an external signal to synchronize its sampling with other SAMUs, or with external time references. The preferred one is the Precision Time Protocol (PTP), also known as 1588; however, the PPS is an accepted alternative. Thanks to its internal high-frequency clock, a SAMU is capable of attributing to each sample a time stamp aligned with the synchronization signal. Considering the importance of the synchronization process, Ref. [44] dedicates several sections to time accuracy tests and to the explanation of how to evaluate and correct potential delays and loss of synchronization. Even the literature is quite vivid in this perspective, and, as it has been described in [45,46], researchers always try to find ways of minimizing delay errors and synchronization issues.

The digitized output of the SAMU is then transmitted through a local data network in the form of data packets containing sampled values (SV), according to [43], and then formatted with the information of voltages, currents, and time. In this way, the receiver is capable of distinguishing and chronologically listing all the data coming from a single SAMU or multiple SAMUs, before further processing them. 


\subsection{Accuracy Evaluation}

The last aspect to be considered when dealing with SAMUs is the metrological one. In particular, this new device may alter or decrease the accuracy of its input signals. Therefore, a SAMU's accuracy has to be standardized and evaluated. From this perspective, Ref. [42] provides tables with accuracy classes (ACs) and their limits for the current and voltage input channels (separately). In addition, the standard dedicates an entire annex to the evaluation of the combined uncertainty resulting from the entire measurement chain of IT plus SAMU. It also specifies the ACs and the limits for such combined uncertainty.

As for the testing of SAMU accuracy, which is more complex due to the digital output, Ref. [42] completely relies on [44], and does not specify other details regarding the accuracy tests. In brief, Annex 9D of [44] provides an example of a test circuit to evaluate devices with a digital output, in which a common clock signal is given to the reference and to the device under test. Afterwards, the two output signals are compared through the use of a composite error that contains information on the phase displacement and the ratio error.

\subsection{Proposed SAMU}

In light of the above, in this work, a new idea of a SAMU is developed, and it consists of (i) an OPAL simulator, being the processing unit of the SAMU; (ii) a resistive divider for the voltage input; and (iii) a shunt-plus-amplifier system for the current input. The developed SAMU accepts both IT and LPITs inputs; in particular, the $100 / \sqrt{3}$ voltage level and $3.25 / \sqrt{3}$, respectively (in the latter case, there is no need for a voltage divider). As for the current inputs, again, the SAMU accepts both the standardized input $1 \mathrm{~A}$ and 5 A for the legacy CTs, and the millivolt input signals from the current LPITs. Finally, OPAL features an IEC 61850-9-2 communication tool that allows us to convert the digital stream of acquired data into the standardized "packets" to be sent to a generic control room or receiver.

In what follows, the description is focused on the single-phase solution SAMU for the sake of simplicity. However, all the results are valid and can be extended to the three-phase solution or even more (16 analogue input channels are available).

Of course, the developed SAMU is just a way to exploit existing equipment to enhance their measurement capabilities and performance. It is not something that aims at replacing existing SAMUs, or at being the unique solution for developing a SAMU.

\section{Measurement Setup}

In this section, the equipment constituting the proposed SAMU is described, together with the instrumentation involved in its characterization process. What follows is structured as the following: Section 3.1 contains the characteristics of the components implemented to build the SAMU; in particular, an OPAL simulator, a voltage divider, and a current shunt are presented. In Section 3.2, the characterization setup is introduced, highlighting the metrological performance of its components.

\subsection{SAMU}

The main idea of this work was to develop a different SAMU, which includes a real time simulator capable of performing HIL measurements, and conditioning devices to adapt the ITs and LPITs outputs for the SAMU input channels. As it is described in the following, the core of the SAMU is based on OPAL technology, whereas the voltage and current signals are addressed by a resistive voltage divider and a resistive shunt, respectively.

\subsubsection{OPAL}

Starting from the processing unit of the developed SAMU, the OPAL-RT OP 4510 Simulator was a Real-Time Simulator (RTS) dedicated to performing real-time simulations and HIL applications using RT-LAB software. The main components constituting the 
RTS used are presented in Figure 2. The color code used in the figure is valid for all the following ones given in this work: blue, device boundaries; green, internal components; yellow, I/O interfaces or available connections.

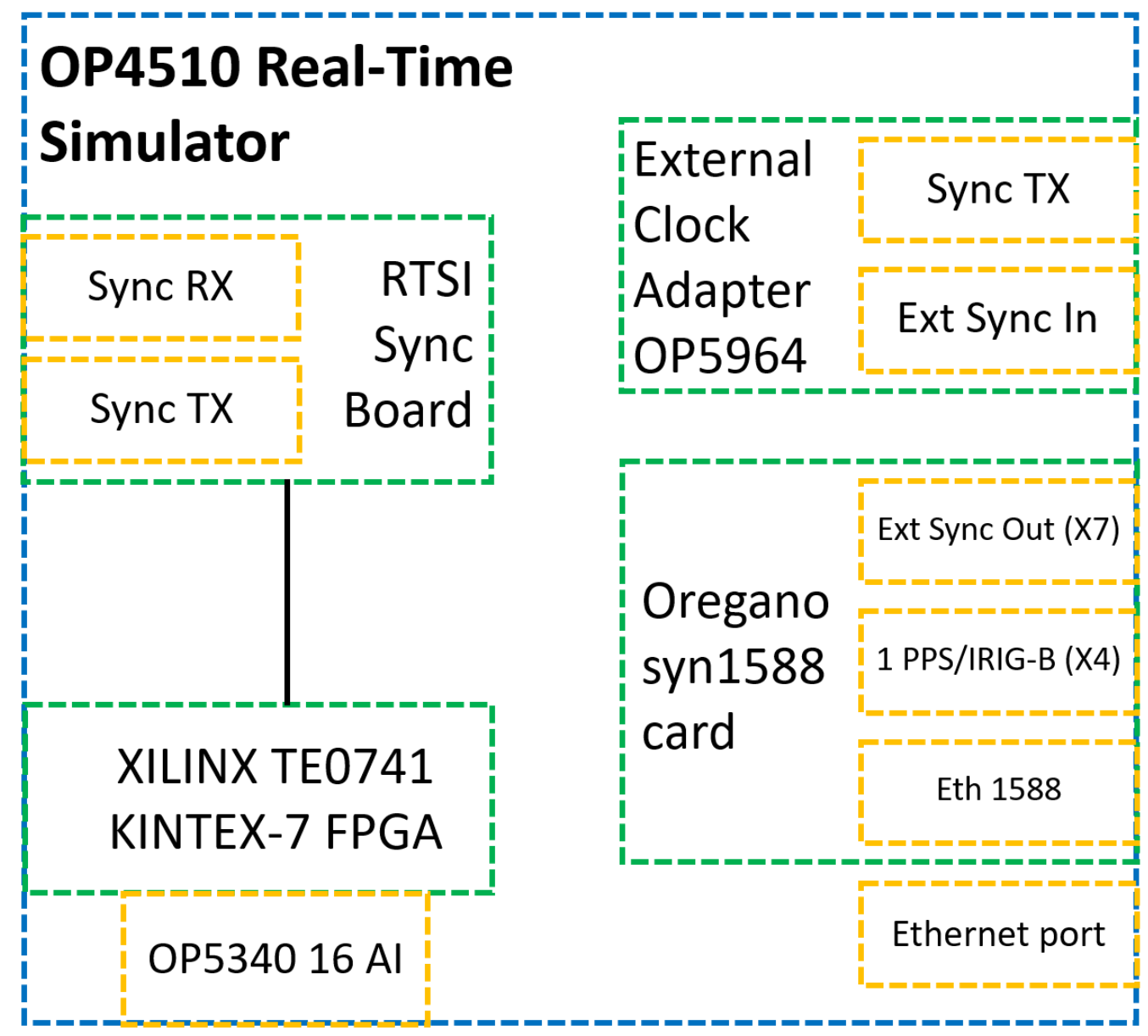

Figure 2. Main components constituting OPAL OP4510 RTS.

The main components included in the considered RTS are:

- Oregano syn1588 PCIe NIC. It is a PCI Express Ethernet network interface with enhancements to provide highly accurate clock synchronization via the IEEE 1588 Standard. In our case, a high-precision OCXO oscillator (Oven Controlled Crystal Oscillator) was installed, and it featured a high accuracy, better than $0.05 \mathrm{ppm}$, for master operations or high accuracy applications. The Oregano card provided all real-time functions required for an IEEE 1588 PTP node to operate, both in master and in slave node, as it could synchronize other devices via the Eth 1588 port shown in Figure 2. This card also allowed us to create time-stamped digital data packets to be transmitted over the network using the same Eth 1588 port. The 1 PPS/IRIGB X4 input port (SMA cable) allowed us to synchronize the Oregano board with either a PPS signal or an IRIG-B signal, coming from a GPS source. The maximum applicable voltage was $3.3 \mathrm{~V}$. The Ext Synch Out X7 port (SMA cable) made available the synchronizing signal to be used by an external board.

- External Clock Adapter OP5964. This module was designated to convert a clock signal from the Oregano syn1588 board into a model synchronization standard compatible with OPAL RTS. The OP5964 could receive the external synchronization signal on the dedicated IO port and it could transmit the synchronizing signal to the RTSI interface from the TX output. 
- $\quad$ RTSI (Real-Time System Interface) Synchronization Board. The synchronization connector was present in the front interface of the RTS. TX and RX allowed, respectively, for the transmission and reception of synchronization data. This board was internally connected to the FPGA.

- XILINX TE0741 KINTEX-7 FPGA. It could accept four standard OPAL-RT mezzanine boards ( 2 digital IO and 2 analog IO boards), in addition to RS422 signals. It offered two types of synchronization, either LVDS or fiber optic, as it was connected to the RTSI interface.

- Analog input card OP5340. It featured 16 differential analog input channels, with a maximum voltage range of $\pm 20 \mathrm{~V}$, sampled at $500 \mathrm{kSa} / \mathrm{s}$. The ADC had a 16-bit resolution, and the minimum acquisition time was $2.5 \mu$ s per channel. The sampling was performed synchronously on all channels by the FPGA; nonetheless, the delay between two channels was evaluated for the sake of completeness in the characterization framework of the entire setup. The declared maximum noise of the analog card was $20 \mathrm{mV}$ peak-to-peak.

- OP4510 RTS also featured an IEC 61850-9-2 LE protocol for digital output data. This protocol allowed us to retrieve 80 or 256 samples per cycle, corresponding to 4000 and 12,800 samples, respectively, at a rated power system frequency of $50 \mathrm{~Hz}$. Using an external clock, the Sample Value (SV) timings were synchronized with an external source, namely the Oregano board, which synchronized the protocol timings according to a specific standard.

- Ethernet port. It was used to interface a laptop to the OP4510 RTS. All the controls were performed in real-time using RT-LAB software.

\subsubsection{Instrument Transformers}

In order to measure the standardized rated voltage and current, a voltage divider was installed on the voltage circuit, whereas a shunt and an amplifier were placed in the current circuit. The devices characteristics were as follows:

- Active voltage divider. It was a resistive voltage divider providing an attenuation ratio of 11.003019 , with a standard deviation of the mean of $1 \cdot 10^{-6}$ at $55 \mathrm{~V}$. It needed to be supplied by an external voltage source at $\pm 15 \mathrm{~V}$.

- Resistive shunt. It was a $10 \mathrm{~A}, 10 \mathrm{~m} \Omega$ shunt. The ratio between the reference and the measured current was 1.000380 , with a standard deviation of the mean $3 \cdot 10^{-6}$ at $5 \mathrm{~A}$.

- Custom amplifier. It featured various multiplication factors, but x100 was the one considered for all the tests described in this work. The amplifier required an external voltage source provided by the $\pm 15 \mathrm{~V}$ voltage supply.

- Low-voltage source. It was a custom $230 / 15 \mathrm{~V}$ voltage supply; it provided $\pm 15 \mathrm{~V}$ and it was used to supply both the voltage divider and the amplifier.

\subsection{Characterization Setup}

Having presented the components constituting the SAMU, its characterization and the characterization of its single components are presented in this section. The required reference devices for the characterization are as follows:

- Arbitrary waveform generator Keysight 33500B. It was used to recreate the PPS signal; therefore, only the specifications concerning the square wave were reported. The rise and fall times were fixed at $8.4 \mathrm{~ns}$, with a resolution of $100 \mathrm{ps}$. The overshoot was typically below $2 \%$ and the duty cycle resolution was $0.01 \%$. The pulse could have a minimum width of $16 \mathrm{~ns}$ with $100 \mathrm{ps}$ resolution. The standard jitter measured with an external source analyzer, at a room temperature of $23^{\circ} \mathrm{C}$, was below $40 \mathrm{ps}$.

- Arbitrary waveform generator RIGOL DG 1022. It was used to generate the $50 \mathrm{~Hz}$ sine waves for a range of amplitudes. The harmonic distortion of a sine wave was $-45 \mathrm{dBc}$ and the phase noise was $-108 \mathrm{dBc} / \mathrm{Hz}$.

- Calibrator Fluke 6105A. In Tables 1-5, the voltage and current sine and harmonics specifications are provided, together with the power specifications. 
- NI-9239 Data AcQuisition Board (DAQ). It was used to separately characterize the sensors adopted to develop the SAMU. The DAQ featured a $\pm 10 \mathrm{~V}$ voltage range and a maximum $50 \mathrm{kSa} / \mathrm{s}$ sampling frequency. Its accuracy features were as follows: $\pm 0.03 \%$ gain error and $\pm 0.01 \%$ offset error.

Table 1. Calibrator voltage specifications.

\begin{tabular}{cccc}
\hline & $\begin{array}{c}\text { Voltage Range } \\
{[\mathbf{V}]}\end{array}$ & $\begin{array}{c}\text { Frequency Range } \\
{[\mathbf{H z}]}\end{array}$ & $\begin{array}{c}\text { Accuracy } \\
\text { [ppm of Output } \pm \mathbf{~ m V} \text { ] }\end{array}$ \\
\hline $\begin{array}{c}\text { Voltage sine } \\
\text { amplitude specs }\end{array}$ & $6.3-90$ & $45-65$ & $42 \pm 0$ \\
& & $16-850$ & $58 \pm 1$ \\
Voltage harmonic & $1.0-23$ & $850-6000$ & $451 \pm 1$ \\
amplitude specs & $6.3-90$ & $16-850$ & $60 \pm 2.2$ \\
& & $850-6000$ & $451 \pm 2.2$ \\
\hline
\end{tabular}

Table 2. Calibrator voltage from the current terminals specifications.

\begin{tabular}{cccc}
\hline & $\begin{array}{c}\text { Voltage Range } \\
{[\mathbf{V}]}\end{array}$ & $\begin{array}{c}\text { Frequency Range } \\
{[\mathbf{H z}]}\end{array}$ & $\begin{array}{c}\text { Accuracy } \\
\text { [ppm of Output } \pm \mu \mathbf{V}]\end{array}$ \\
\hline Voltage sine & $0.05-0.25$ & & $73 \pm 10$ \\
Amplitude specs & $0.6-1.5$ & $45-65$ & $53 \pm 50$ \\
& $4-10$ & & $52 \pm 200$ \\
\hline
\end{tabular}

Table 3. Calibrator current specifications.

\begin{tabular}{cccc}
\hline & $\begin{array}{c}\text { Current Range } \\
{[\mathbf{A}]}\end{array}$ & $\begin{array}{c}\text { Frequency Range } \\
{[\mathbf{H z}]}\end{array}$ & $\begin{array}{c}\text { Accuracy } \\
\text { [ppm of Output } \pm \mu \mathbf{A}]\end{array}$ \\
\hline Current sine & $0.1-0.25$ & & $46 \pm 1.5$ \\
Amplitude specs & $0.4-1.0$ & $45-65$ & $47 \pm 6$ \\
Current harmonic & $2-5$ & & $49 \pm 30$ \\
Amplitude specs & $1-10$ & $16-850$ & $64 \pm 200$ \\
\hline
\end{tabular}

Table 4. Calibrator current to voltage phase specifications.

\begin{tabular}{cccc}
\hline $\begin{array}{c}\text { Voltage Range } \\
{[\mathrm{V}]}\end{array}$ & $\begin{array}{c}\text { Current Range } \\
{[\mathrm{A}]}\end{array}$ & $\begin{array}{c}\text { Frequency Range } \\
{[\mathrm{Hz}]}\end{array}$ & $\begin{array}{c}\text { Accuracy } \\
{\left[{ }^{\circ} \pm \mathbf{5}^{\circ}\right]}\end{array}$ \\
\hline $23-1008$ & $0.25-21$ & $45-65$ & 0.0023 \\
\hline
\end{tabular}

Table 5. Calibrator power specifications.

\begin{tabular}{|c|c|c|c|c|c|}
\hline $\begin{array}{c}\text { Frequency Range } \\
{[\mathrm{Hz}]}\end{array}$ & $\begin{array}{c}\text { Voltage Range } \\
\text { [V] }\end{array}$ & $\begin{array}{l}\text { Power } \\
\text { Factor }\end{array}$ & $\begin{array}{c}\text { Current Range } \\
{[\mathrm{A}]}\end{array}$ & $\begin{array}{c}\text { Power with Current at } \\
50 \% \text { of Range } \\
\text { [ppm] }\end{array}$ & 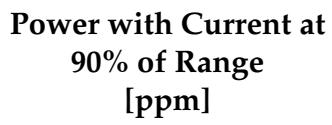 \\
\hline \multirow{3}{*}{$45-65$} & \multirow{3}{*}{$23-90$} & \multirow[b]{2}{*}{1.0} & $0-2$ & 72 & 62 \\
\hline & & & $5-50$ & 74 & 65 \\
\hline & & 0.5 & $0-5$ & 100 & 93 \\
\hline
\end{tabular}

\section{Characterization Procedure}

In this section, a detailed description of the characterization procedure is provided. In the Section 4.1, the OPAL characterization setup is presented. This is followed by the sensors' characterization setup in 4.2 and, finally, the whole SAMU test arrangement 
is illustrated in 4.3. The aim is to start with the characterization of the single elements composing the whole SAMU in order to understand the single contributors to the overall SAMU accuracy.

\subsection{OPAL Characterization}

In this section, the tests of amplitude, the phase delay introduced by OPAL, and the phase delay between two input channels of OPAL setups are, respectively, presented in Section 4.1.1, Section 4.1.2, and Section 4.1.3. In addition, in Section 4.1.4, the OPAL accuracy performances are tested with several distorted signals.

This set of tests was fundamental for evaluating the accuracy of the RTS, before assessing the whole setup representing the SAMU solution.

\subsubsection{Amplitude Characterization}

The setup used for the amplitude characterization is presented in Figure 3.

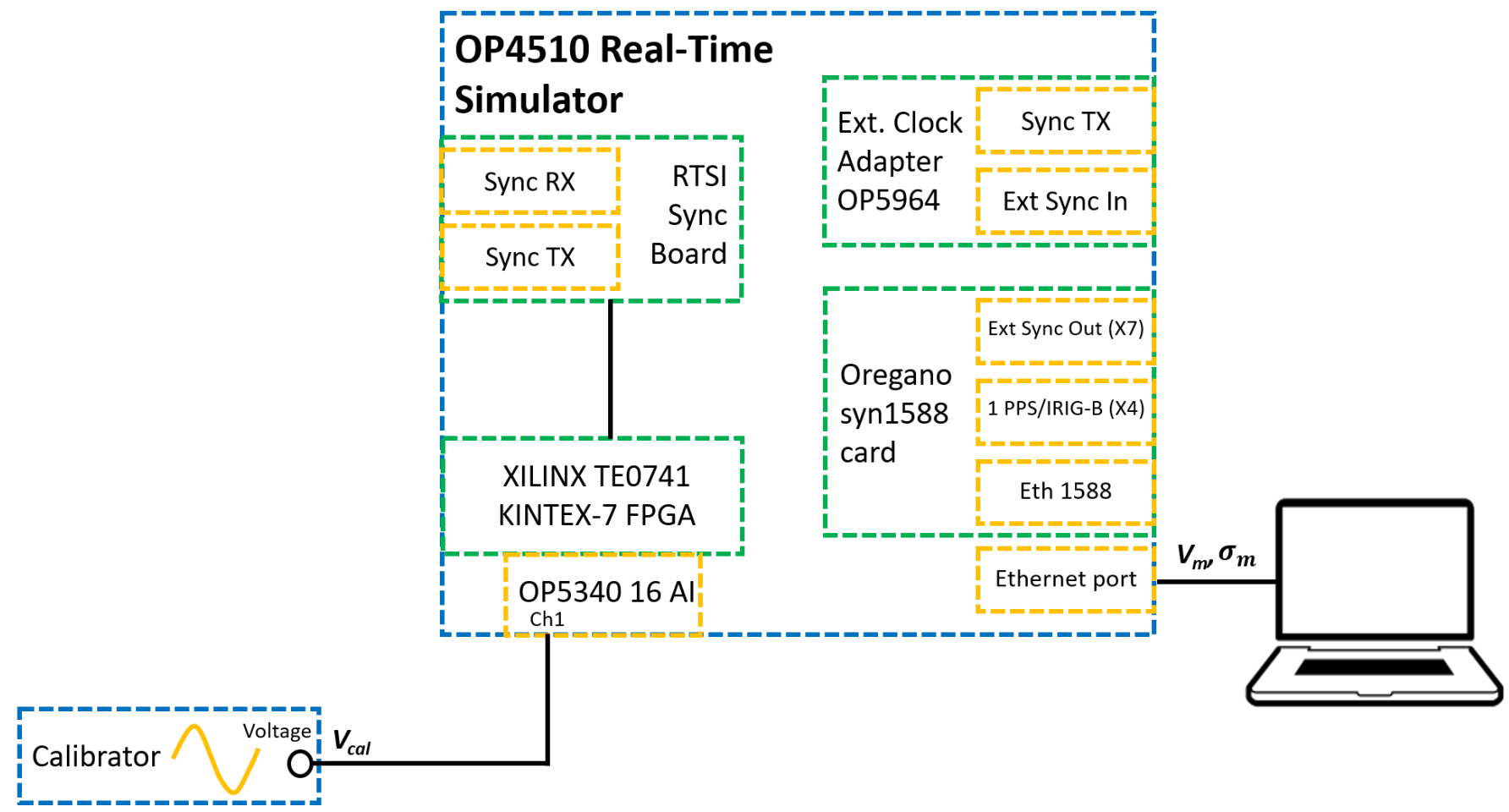

Figure 3. Setup used for amplitude characterization of OPAL RTS with a Fluke 6105A Calibrator.

The aim of this test was to assess the RTS accuracy in terms of amplitude. Therefore, the voltage levels $\left(V_{\text {cal }}\right)$ listed in Table 6 were directly applied to the RTS with the reference voltage source (Fluke $6105 \mathrm{~A}$ ). For each voltage level, 500 measurements of the RMS value were performed.

Table 6. Voltages applied from the Fluke 6105A Calibrator $\left(V_{\text {cal }}\right)$ to the RTS in [V] at $50 \mathrm{~Hz}$.

\begin{tabular}{cc}
\hline$V_{c a l}[\mathbf{V}]$ \\
\hline 0.010 \\
0.050 \\
0.500 \\
1 \\
5 \\
7 \\
10 \\
\hline
\end{tabular}




\subsubsection{Phase Displacement}

The setup used for the evaluation of phase displacement, introduced by the RTS, is presented in Figure 4.

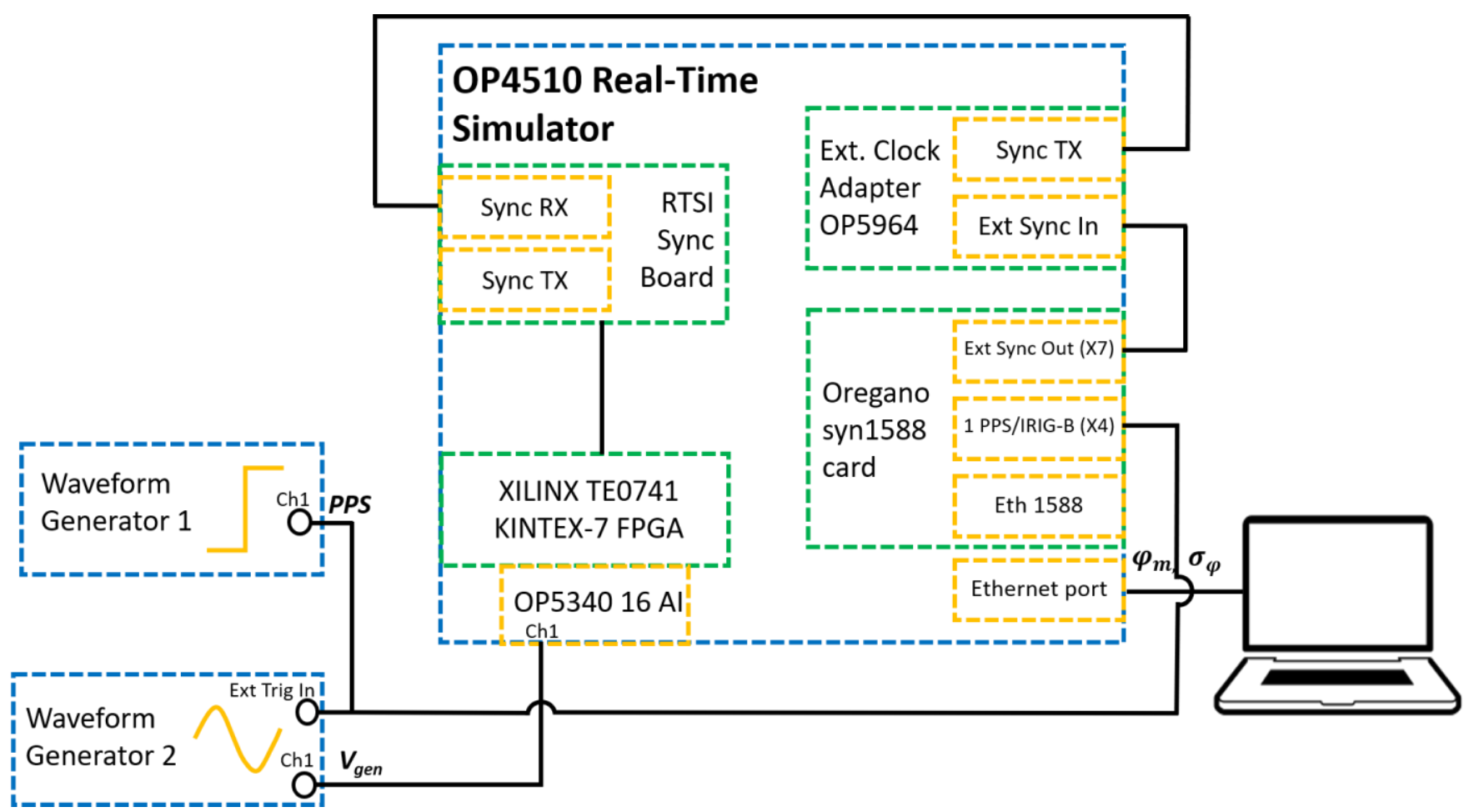

Figure 4. Setup used for phase delay characterization of OPAL RTS.

The objective of this test was to assess phase displacement and its corresponding time delay, introduced by the processing unit, with respect to the synchronization signal (PPS in this case). In fact, as detailed in Section 2, the synchronization signal started the acquisition of the data coming from the ITs outputs.

For this investigation, two arbitrary waveform generators were used, namely Keysight 33500B and RIGOL DG 1022, corresponding, respectively, to Waveform Generator 1 (WG1) and Waveform Generator 2 (WG2) in Figure 4.

WG1 was used to generate a square wave with a $3.3 \mathrm{~V}$ amplitude, an offset of $1.65 \mathrm{~V}$, and a period of $1 \mathrm{~Hz}$, corresponding to $1 \mathrm{~s}$. The duty cycle was set to $50 \%$. This square wave replicated the 1 PPS source one could obtain from a GPS antenna, and it was sent to the PPS input channel of OPAL (SMA connector X4 of the Oregano card) and to the external trigger input of WG2. This device was configured to be working in burst mode, and it was set to generate, without any additional delay, 20 cycles of a $50 \mathrm{~Hz}$ voltage sinusoid for different RMS values, presented in Table 7.

Table 7. Voltages applied from the WG2 $\left(V_{g e n}\right)$ to the RTS in [V] at $50 \mathrm{~Hz}$.

\begin{tabular}{c}
\hline$V_{\text {gen }}[\mathrm{V}]$ \\
0.010 \\
0.050 \\
0.500 \\
1 \\
5 \\
7
\end{tabular}


The output voltage of WG2 was acquired with the analog card of the RTS. The choice of the roles of WG1 and WG2 was made based on the fact that WG1 has a better jitter with respect to WG2 for square wave generation.

What played a major role in this setup was the Oregano syn1588 card. This device was configured to work as a PTP Master using a 1 PPS signal, as it could receive either 1 PPS or an IRIG-B signal from a GPS antenna. The board was also set up to wait before the next second. It meant that the beginning of the simulation was delayed until the next second change. In this way, the rising edge of the synchronization signal was aligned to the rising edge of the 1 PPS signal, as defined by [43].

Another important setting was the synchronization timeout. This parameter, if set equal to zero, indicated that the simulation started immediately after the model was loaded. Generally, it could be increased in such a way that the simulation started with good synchronization accuracy with the external source. According to the datasheet, if the Oregano board was synchronized by a good IRIG-B or 1 PPS signal, the accuracy could reach 25 to $100 \mathrm{~ns}$.

The synchronization accuracy took into account the accuracy between the time step of the simulation and the 1 PPS. When synchronizing with a 1 PPS signal from an external clock, the Oregano board would send, in any case, a synchronizing pulse to the FPGA at each time step of the model ( $20 \mu$ in our case). The 1 PPS from the external clock would be used for some micro-adjustment to the clocking, to be sure that it was actually synchronized at the second.

Given this rationale, the external trigger out (SMA connector X7) of the Oregano board provided an external synchronization signal, which was sent to the Clock Adapter OP5964 entering the External Signal in the SMA connector. The Clock Adapter role has been previously described, and it was needed to convert the clock signal from the Oregano board into a model synchronization standard compatible with OPAL RTS. For this reason, the synchronizing signal was transmitted from the Sync TX jack to the Sync RX receiver in the RTSI board via an audio cable. The RTSI synchronization board was internally connected to the FPGA; therefore, it could propagate the synchronizing signal to the FPGA and perform the micro-adjustments explained before.

The described setup was used to perform 100 measurements for each of the sine voltages listed in Table 7.

\subsubsection{Phase Displacement between Two Channels}

The setup used for the characterization of the phase displacement between two analog input channels of OPAL RTS is illustrated in Figure 5.

As can be perceived from Figure 5, the idea behind this setup was exactly the same as the one described in the aforementioned configuration in Section 4.1.2.

Compared to the previous setup, in this case, the output of WG2 was sent to two different analog input channels of the analog card of OPAL RTS.

Despite the fact that the acquisition in the RTS was performed synchronously by the FPGA, in this test, the objective was actually to measure the time delay between two analog voltage sine waves triggered by an external PPS generated by the WG1, as before.

Table 7 previously described holds; also, in this case, 100 measurements were carried out for each of the listed signals, and a synchronization accuracy of 25 ns was kept constant for the entire duration of the tests.

\subsubsection{Distorted Signals Characterization}

Given the increasing presence of superimposed harmonics on voltages and currents in the power system, this section describes the characterization of the RTS behavior vs. the distorted signals directly applied from the Fluke 6105 reference.

The measurement setup used for this test was the same as the one depicted in Figure 3. The tests consisted of applying, to the RTS input, a $5 \mathrm{~V}$ signal at $50 \mathrm{~Hz}$, superimposed with a mix of harmonics from the 3rd to the 9th. The harmonics and their amplitudes, used to 
generate the distorted signals, were selected in accordance with EN 50160 [47]. For each distorted signal applied to the RTS, 500 measurements of THD were performed and compared to the THD set on the reference calibrator. Analogously, a distorted signal, gathered from in-field measurements performed during a fault and containing harmonics up to the 29th, was used as a further test signal and evaluated again with the THD parameter.

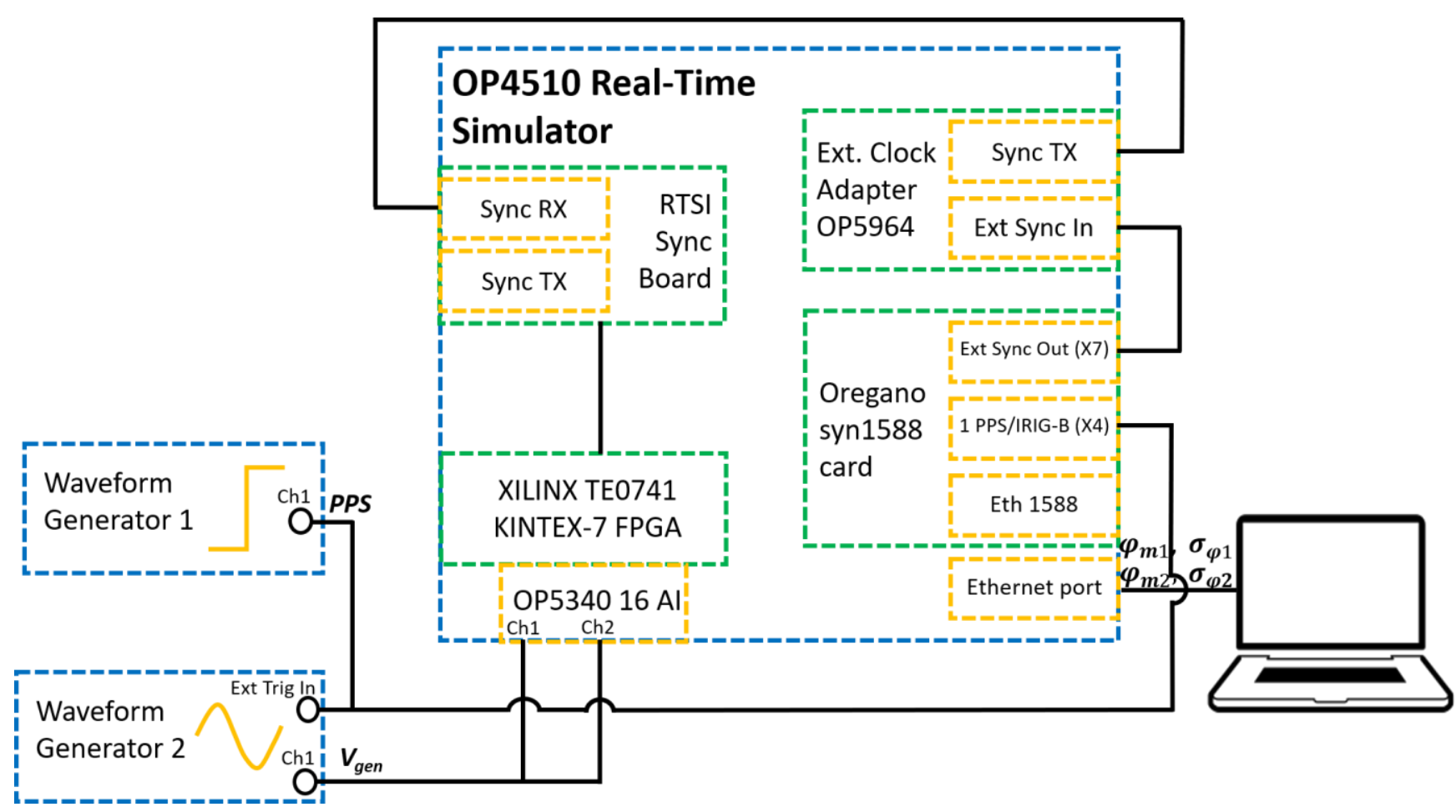

Figure 5. Setup used for phase delay characterization between two analog input channels of OPAL RTS.

\subsection{Sensors Characterization}

Once the RTS is characterized, the remaining components to be assessed are the voltage and current sensors. Therefore, this section describes the characterization setup and procedure that allowed us to evaluate the accuracy of the adopted sensors. Figure $6 \mathrm{a}$ shows the measurement setup used to characterize the voltage sensor, while Figure $6 \mathrm{~b}$ shows the one used for the current sensor, plus amplifier.

An important remark worth underlining, when looking at Figure 6, is that the characterization process included the DAQ described in Section 3.2. However, given the characteristics of the DAQ board, it is clear that its contribution to the total uncertainty was negligible.

\subsection{SAMU Characterization}

The characterization process concluded with the assessment of the metrological performance of the whole SAMU developed in this work. Figure 7 depicts the adopted measurement setup. It includes only components that have already been described.

\subsubsection{Amplitude Characterization}

This first set of tests involved the assessment of the amplitude measured by the SAMU considering the complete measurement chain sensor + RTS. Therefore, $80 \%, 100 \%$, and $120 \%$ of the rated voltage $(57 \mathrm{~V})$, and $5 \%, 20 \%$, and $100 \%$ of the rated current $(5 \mathrm{~A})$, were applied/injected with the Fluke $6105 \mathrm{~A}$. Afterwards, 500 measurements of the RMS value of the voltages and currents measured by the SAMU were collected and assessed. 


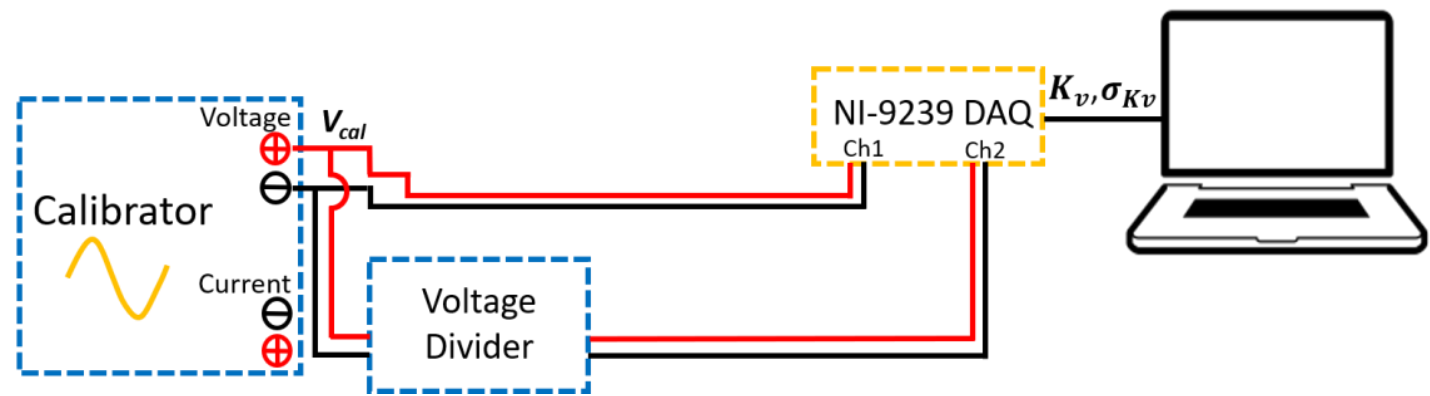

(a)

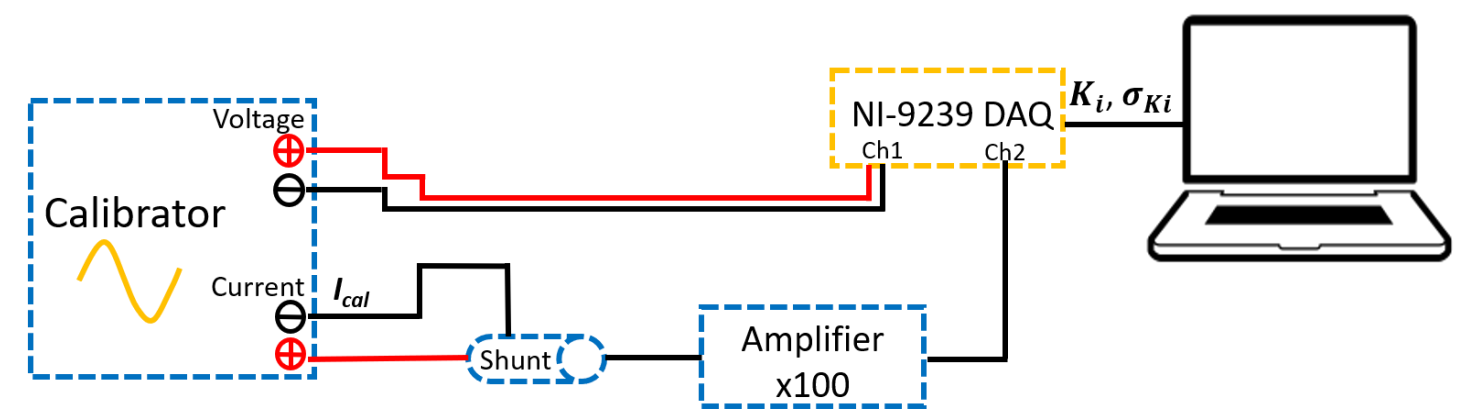

(b)

Figure 6. Sensors' characterization setups: (a) voltage divider setup for the voltage branch; (b) resistive shunt and amplifier setup for the current branch.

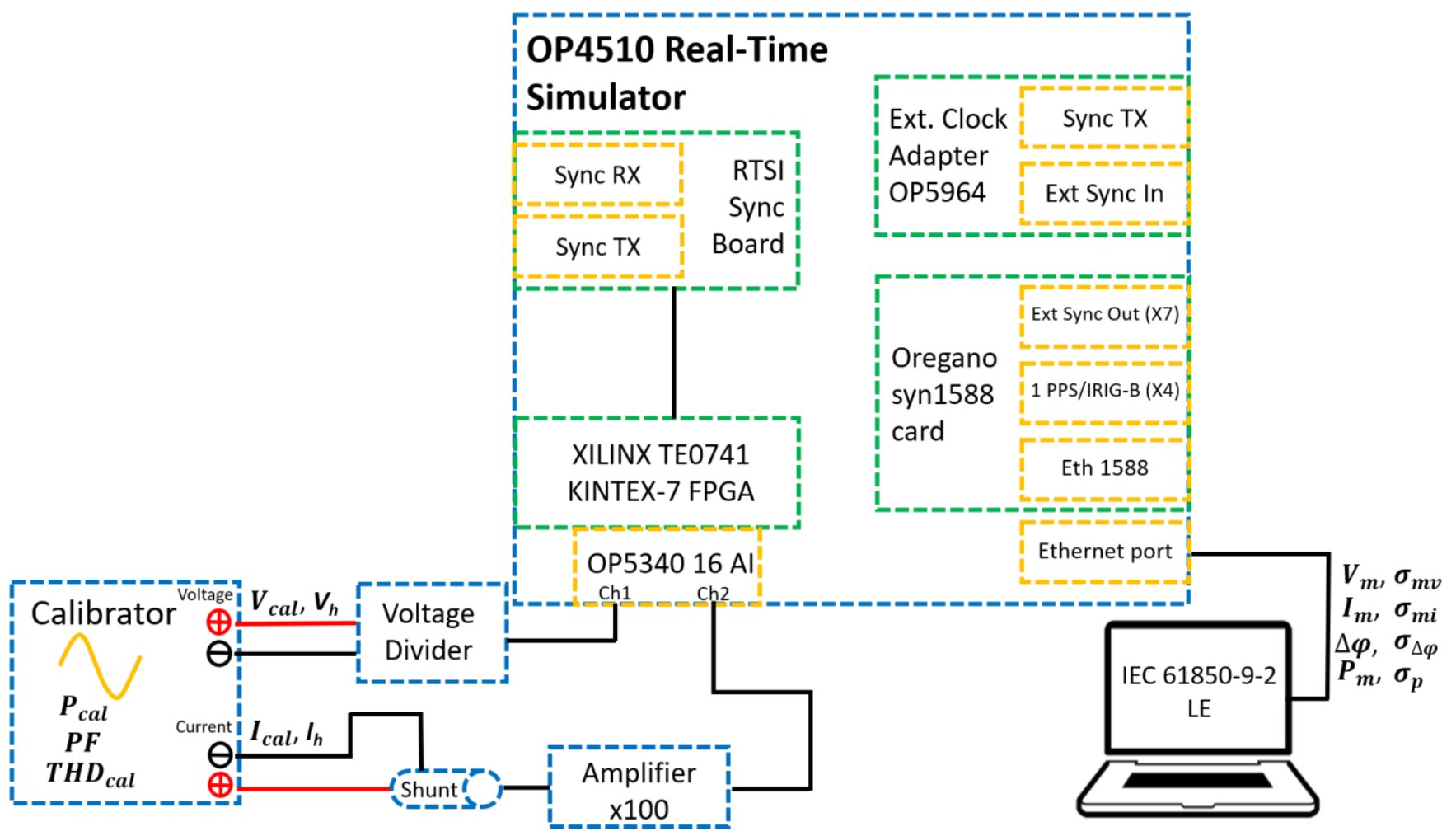

Figure 7. Final setup of the realized SAMU acquiring voltages and currents from the Fluke 6105A Calibrator and OPAL RTS, providing data using the IEC 61850-9-2 LE protocol. 


\subsubsection{Phase Characterization}

Considering all the voltage and current values combination described in Section 4.3.1, the phase difference between the voltage and current channels was measured. The phase difference between the voltage and current was set to zero on the Fluke 6105, and 500 measurements were collected and evaluated.

\subsubsection{Characterization Using Distorted Signals}

In accordance with what was described in Section 4.1.4, for the simple RTS characterization, the whole SAMU was tested by applying/injecting distorted signals. Such signals were those introduced in Section 4.1.4, with the difference being that the rated voltage was not $5 \mathrm{~V}$ but $57 \mathrm{~V}$ for the voltage channel, and $5 \mathrm{~A}$ for the current one. Consequently, the harmonic amplitude percentages were calculated starting from those two values.

\subsubsection{Power Characterization}

This last set of tests aimed at extending the capabilities of a SAMU. Of course, the standard does not prescribe SAMUs for being capable of computing powers or energy. However, considering the computational capabilities of the developed SAMU, this further test added the wattmeter feature. Furthermore, considering the simultaneous presence of voltages and currents and the SAMU inputs, it might be useful to exploit its digital output to send power information to the control room, or to the data collector.

By using the setup depicted in Figure 7, 500 measurements of the following test were performed:

- Test at rated conditions. The voltage was set to $57 \mathrm{~V}$ and the current to $5 \mathrm{~A} \mathrm{(at} 50 \mathrm{~Hz}$ ).

- Test varying the amplitudes. From the combination of $80 \%, 100 \%$, and $120 \%$ of the rated voltage and $5 \%, 20 \%$, and $100 \%$ of the rated current, 9 power values were applied and measured (always at $50 \mathrm{~Hz}$ ).

- Test varying the power factor (PF). At rated voltage and current, the PF was set, varying from 1.0 to 0.1 at 0.1 steps.

\section{Results and Discussion}

This section includes all the results obtained from the sets of tests described in Section 4 .

\subsection{OPAL Characterization Results}

\subsubsection{Amplitude Test Results}

Table 8 reports the amplitude test results in terms of mean values of 500 measurements. The table shows the RMS voltage applied from the calibrator $\left(V_{c a l}\right)$, the measured voltage $\left(V_{m}\right)$ and its standard deviation of the mean $\left(\sigma_{m}\right)$, and the relative error $\varepsilon_{r}$ between $V_{m}$ and $V_{\text {cal }}$ computed as:

$$
\varepsilon_{r}=\frac{V_{m}-V_{c a l}}{V_{c a l}} \cdot 100 .
$$

Table 8. Results obtained from the amplitude characterization tests of OPAL RTS with a Fluke 6105A Calibrator.

\begin{tabular}{ccccc}
\hline $\begin{array}{c}V_{\text {cal }} \\
{[\mathbf{V}]}\end{array}$ & $\begin{array}{c}\boldsymbol{V}_{m} \\
{[\mathbf{V}]}\end{array}$ & $\begin{array}{c}\sigma_{m} \\
{[\mathbf{V}]}\end{array}$ & $\begin{array}{c}\mathcal{E}_{r} \\
{[\%]}\end{array}$ & $\begin{array}{c}\sigma_{\varepsilon v} \\
{[\%]}\end{array}$ \\
\hline 0.010 & 0.010027 & $4 \cdot 10^{-6}$ & 0.50 & $2 \cdot 10^{-2}$ \\
0.050 & 0.049157 & $5 \cdot 10^{-6}$ & 1.685 & $6 \cdot 10^{-3}$ \\
0.500 & 0.499069 & $4 \cdot 10^{-6}$ & 0.1862 & $5 \cdot 10^{-4}$ \\
1 & 0.999133 & $3 \cdot 10^{-6}$ & 0.0867 & $2 \cdot 10^{-4}$ \\
5 & 4.997667 & $2 \cdot 10^{-6}$ & 0.04666 & $3 \cdot 10^{-5}$ \\
7 & 7.000031 & $3 \cdot 10^{-6}$ & 0.04429 & $3 \cdot 10^{-5}$ \\
10 & 9.995478 & $5 \cdot 10^{-6}$ & 0.04522 & $4 \cdot 10^{-5}$ \\
\hline
\end{tabular}


The last column of Table 8 reports the standard deviation of the mean of $\varepsilon_{r}$, referred to as $\sigma_{\varepsilon v}$.

From Table 8 , it is clear that measurements below $50 \mathrm{mV}$ are strongly affected by $20 \mathrm{mV}$ peak-to-peak noise, declared in the datasheet of the device. On the contrary, for higher voltages, $\varepsilon_{r}$ is always below two parts per thousand. In addition, from the values of $\sigma_{\varepsilon v}$, it is possible to appreciate the degree of accuracy that can be reached with the RTS.

\subsubsection{Phase Displacement Test Results}

Table 9 reports the phase displacement test results in terms of mean value of 100 measurements. The table contains the RMS voltage applied from the WG2 $\left(V_{g e n}\right)$, the measured phase $\left(\varphi_{m}\right)$, and its standard deviation of the mean $\left(\sigma_{\varphi}\right)$.

Table 9. Results obtained from the phase characterization tests of OPAL RTS, with WG1 generating the 1 PPS square wave and WG2 generating the voltage sine wave at $50 \mathrm{~Hz}$.

\begin{tabular}{ccccc}
\hline $\begin{array}{c}V_{\text {gen }} \\
{[\mathbf{V}]}\end{array}$ & $\begin{array}{c}\boldsymbol{\varphi}_{\boldsymbol{m}} \\
{[\mathbf{r a d}]}\end{array}$ & $\begin{array}{c}\sigma_{\boldsymbol{\varphi}} \\
{[\mathbf{r a d}]}\end{array}$ & $\begin{array}{c}\boldsymbol{t}_{\boldsymbol{m}} \\
{[\mathbf{s}]}\end{array}$ & $\begin{array}{c}\sigma_{t} \\
{[\mathrm{~s}]}\end{array}$ \\
\hline 0.010 & -0.00480 & $9 \cdot 10^{-5}$ & $-1.53 \cdot 10^{-5}$ & $3 \cdot 10^{-7}$ \\
0.050 & -0.00466 & $4 \cdot 10^{-5}$ & $-1.48 \cdot 10^{-5}$ & $1 \cdot 10^{-7}$ \\
0.500 & -0.00488 & $4 \cdot 10^{-5}$ & $-1.55 \cdot 10^{-5}$ & $1 \cdot 10^{-7}$ \\
1 & -0.00493 & $4 \cdot 10^{-5}$ & $-1.57 \cdot 10^{-5}$ & $1 \cdot 10^{-7}$ \\
5 & -0.00490 & $4 \cdot 10^{-5}$ & $-1.56 \cdot 10^{-5}$ & $1 \cdot 10^{-7}$ \\
7 & -0.00490 & $4 \cdot 10^{-5}$ & $-1.56 \cdot 10^{-5}$ & $1 \cdot 10^{-7}$ \\
\hline
\end{tabular}

For the sake of completeness, the corresponding time delay with respect to a $50 \mathrm{~Hz}$ sine wave is also reported $\left(t_{m}\right)$, together with its standard deviation of the mean $\left(\sigma_{t}\right)$.

The whole set of tests is performed with a synchronization accuracy of $25 \mathrm{~ns}$ between the time step of the simulation and the 1 PPS signal, generated by WG1.

The results presented in Table 9 show that the noise level for low-voltage signals do not influence phase displacement as much as when the amplitude is at very low levels. Furthermore, $\varphi_{m}$ remains almost constant, independent of the voltage amplitude adopted for the test.

A final note after presenting the amplitude and phase displacement tests is that both tests were performed on other channels to understand whether they behave in the same way. The results, not reported here for the sake of brevity, confirmed that all channels have coherent behaviors, both in amplitude and in phase.

\subsubsection{Phase Displacement between Two Channels Test Results}

Table 10 reports the results of the phase displacement between two channels test. The results are presented in terms of mean value of 100 measurements. Table 10 includes the RMS voltage applied from the WG2 $\left(V_{g e n}\right)$, the phase measured $\left(\varphi_{m 1}\right)$ on channel 1 and its standard deviation of the mean $\left(\sigma_{\varphi 1}\right)$, and the measured phase $\left(\varphi_{m 2}\right)$ on channel 2 and its standard deviation of the mean $\left(\sigma_{\varphi 2}\right)$. Finally, the last two columns report the phase difference $(\Delta \varphi)$ between channel $1\left(\varphi_{m 1}\right)$ and $2\left(\varphi_{m 2}\right)$, and its associated standard deviation of the mean $\left(\sigma_{\Delta \varphi}\right)$.

From Table 10, it can be observed that $\Delta \varphi$ and its uncertainty are slightly affected by the voltage amplitude used to run the test. In fact, for voltage values below $50 \mathrm{mV}, \Delta \varphi$ is only a fraction of milliradian. On the contrary, for higher voltage values, $\Delta \varphi$ decreases up to a few microradians, with a $\sigma_{\Delta \varphi}$ in the order of $10^{-7}$.

\subsubsection{Distorted Signals Test Results}

Table 11 lists the results of the tests in which the reference calibrator applies distorted voltages directly to the RTS ( $5 \mathrm{~V}$ for the $50 \mathrm{~Hz}$ component). From the table, the five distorted signals, A to E, are listed and described. In particular, $\mathrm{h}$ is the harmonic order, $V_{h}$ is the relative amplitude in [\%] of the $50 \mathrm{~Hz}$ component, $T H D_{\text {cal }}$ is the THD applied by the 
calibrator, and $T H D_{c}$ is the one computed by the RTS. Finally, $d T H D$ is the relative error in $[\%]$ between the two previous values.

Table 10. Results obtained from the phase displacement between two channels of OPAL RTS with a Fluke 6105A Calibrator.

\begin{tabular}{ccccccc}
\hline $\begin{array}{c}V_{g e n} \\
{[\mathbf{V}]}\end{array}$ & $\begin{array}{c}\boldsymbol{\varphi}_{m 1} \\
{[\mathbf{r a d}]}\end{array}$ & $\begin{array}{c}\sigma_{\varphi 1} \\
{[\mathbf{r a d}]}\end{array}$ & $\begin{array}{c}\boldsymbol{\varphi}_{m 2} \\
{[\mathrm{rad}]}\end{array}$ & $\begin{array}{c}\sigma_{\varphi 2} \\
{[\mathbf{r a d}]}\end{array}$ & $\begin{array}{c}\Delta \varphi \\
{[\mathbf{r a d}]}\end{array}$ & $\begin{array}{c}\sigma_{\Delta \varphi} \\
{[\mathrm{rad}]}\end{array}$ \\
\hline 0.010 & -0.0049 & $1 \cdot 10^{-4}$ & -0.0050 & $1 \cdot 10^{-4}$ & $4.4 \cdot 10^{-5}$ & $1 \cdot 10^{-4}$ \\
0.050 & -0.00453 & $4 \cdot 10^{-5}$ & -0.00465 & $4 \cdot 10^{-5}$ & $1.1 \cdot 10^{-4}$ & $2 \cdot 10^{-5}$ \\
0.500 & -0.00486 & $4 \cdot 10^{-5}$ & -0.00487 & $4 \cdot 10^{-5}$ & $9 \cdot 10^{-6}$ & $2 \cdot 10^{-6}$ \\
1 & -0.00492 & $4 \cdot 10^{-5}$ & -0.00493 & $4 \cdot 10^{-5}$ & $1.6 \cdot 10^{-5}$ & $1 \cdot 10^{-6}$ \\
5 & -0.00490 & $4 \cdot 10^{-5}$ & -0.00491 & $4 \cdot 10^{-5}$ & $7.3 \cdot 10^{-6}$ & $2 \cdot 10^{-7}$ \\
7 & -0.00489 & $4 \cdot 10^{-5}$ & -0.00490 & $4 \cdot 10^{-5}$ & $5.4 \cdot 10^{-6}$ & $2 \cdot 10^{-7}$ \\
\hline
\end{tabular}

Table 11. Distorted voltages directly applied to OPAL RTS at at $5 \mathrm{~V}$.

\begin{tabular}{|c|c|c|c|c|c|c|}
\hline Signal & $\begin{array}{c}h \\
{[/]}\end{array}$ & $\begin{array}{c}V_{h} \\
{[\%]}\end{array}$ & $\begin{array}{c}T H D_{c a l} \\
{[\%]}\end{array}$ & $\begin{array}{c}T_{H} D_{c} \\
{[\%]}\end{array}$ & $\begin{array}{l}\sigma_{c} \\
{[/]}\end{array}$ & $\begin{array}{c}d T H D \\
{[\%]}\end{array}$ \\
\hline A & 3 & 5 & 5 & 5.00365 & $5 \cdot 10^{-5}$ & 0.0730 \\
\hline \multirow[t]{2}{*}{ B } & $\begin{array}{l}3 \\
5\end{array}$ & $\begin{array}{l}5 \\
6\end{array}$ & \multirow[t]{2}{*}{7.8} & \multirow[t]{2}{*}{7.83122} & \multirow[t]{2}{*}{$6 \cdot 10^{-5}$} & \multirow[t]{2}{*}{0.4003} \\
\hline & 3 & 5 & & & & \\
\hline \multirow[t]{3}{*}{$\mathrm{C}$} & 5 & 6 & \multirow[t]{3}{*}{9.3} & \multirow[t]{3}{*}{9.31026} & \multirow[t]{3}{*}{$6 \cdot 10^{-5}$} & \multirow[t]{3}{*}{0.1103} \\
\hline & 7 & 5 & & & & \\
\hline & 3 & 5 & & & & \\
\hline \multirow{7}{*}{$\mathrm{D}$} & 5 & 6 & \multirow{7}{*}{9.4} & \multirow{7}{*}{9.43183} & \multirow[t]{7}{*}{$6 \cdot 10^{-5}$} & \multirow[t]{7}{*}{0.3386} \\
\hline & 7 & 5 & & & & \\
\hline & 9 & 1.5 & & & & \\
\hline & 3 & 2 & & & & \\
\hline & 5 & 10 & & & & \\
\hline & 7 & 10 & & & & \\
\hline & 11 & 10 & & & & \\
\hline \multirow[t]{5}{*}{$\mathrm{E}$} & 13 & 3 & \multirow[t]{5}{*}{18.7} & \multirow[t]{5}{*}{18.67570} & \multirow[t]{5}{*}{$6 \cdot 10^{-5}$} & \multirow[t]{5}{*}{0.1299} \\
\hline & 17 & 3 & & & & \\
\hline & 23 & 3 & & & & \\
\hline & 25 & 2 & & & & \\
\hline & 29 & 1.5 & & & & \\
\hline
\end{tabular}

From the table, it is clear that the distorted signals do not affect the measurements taken with the RTS. For all the distorted signals, from A to E, $d T H D$ is always lower than $0.3 \%$. Consequently, it can be concluded that the RTS is suitable and accurate enough to perform power quality evaluations on the input signals.

\subsection{Sensors Characterization Results}

In this section, the results obtained from the characterization of the employed voltage and current sensors, tested according to the setups illustrated in 4.2, are presented.

Table 12 lists the results (100 measurements) of the voltage divider characterization. In the first two columns, the divider ratio, $K_{v}$, is provided, together with its standard deviation of the mean, $\sigma_{K v}$; the next two columns indicate the phase displacement in [rad], introduced by the voltage sensor, $\varphi_{v}$, and its standard deviation of the mean, $\sigma_{\varphi v}$. The last column reports the reference voltage set in the calibrator $\left(V_{\text {cal }}\right)$ in RMS value.

The results obtained in Table 12, specifically the ones related to 45,55 and $70 \mathrm{~V}$ reference voltages, are used to compensate the phase of the measured voltage in [rad] and apply the correct voltage ratio, $K_{v}$, in the next section of tests in 5.3. However, as it can be perceived, the influence of the phase correction is irrelevant. Furthermore, it can be stated that the voltage sensor is stable and accurate. 
Table 12. Voltage divider characterization performed for different values of the reference voltage supplied by the Fluke 6105A Calibrator.

\begin{tabular}{ccccc}
\hline $\begin{array}{c}K_{v} \\
{[/]}\end{array}$ & $\begin{array}{c}\sigma_{K v} \\
{[/]}\end{array}$ & $\begin{array}{c}\varphi_{v} \\
{[\mathbf{r a d}]}\end{array}$ & $\begin{array}{c}\sigma_{\varphi v} \\
{[/]}\end{array}$ & $\begin{array}{c}V_{c a l} \\
{[\mathbf{V}]}\end{array}$ \\
\hline 11.003234 & $1 \cdot 10^{-6}$ & $-3.16 \cdot 10^{-5}$ & $3 \cdot 10^{-7}$ & 40 \\
11.003204 & $1 \cdot 10^{-6}$ & $-3.45 \cdot 10^{-5}$ & $2 \cdot 10^{-7}$ & 45 \\
11.003064 & $1 \cdot 10^{-6}$ & $-3.11 \cdot 10^{-5}$ & $2 \cdot 10^{-7}$ & 50 \\
11.003019 & $1 \cdot 10^{-6}$ & $-3.06 \cdot 10^{-5}$ & $2 \cdot 10^{-7}$ & 55 \\
11.002972 & $1 \cdot 10^{-6}$ & $-2.94 \cdot 10^{-5}$ & $2 \cdot 10^{-7}$ & 60 \\
11.002956 & $1 \cdot 10^{-6}$ & $-2.85 \cdot 10^{-5}$ & $3 \cdot 10^{-7}$ & 65 \\
11.002932 & $1 \cdot 10^{-6}$ & $-2.91 \cdot 10^{-5}$ & $2 \cdot 10^{-7}$ & 70 \\
\hline
\end{tabular}

The second part of the sensors' characterization tests involved the current branch. In this part, the resistive shunt and the custom amplifier were characterized together, as they were used simultaneously. Table 13 presents these results (100 measurements): the ratio between the reference current and the measured current $\left(K_{i}\right)$ is provided, together with its standard deviation of the mean, $\sigma_{K i}$, in the first two columns; the next two, instead, indicate the phase displacement in [rad], introduced by the sensors, $\varphi_{i}$, and its standard deviation of the mean, $\sigma_{\varphi i}$. The last column reports the reference current set in the calibrator $\left(I_{\text {cal }}\right)$ in $[\mathrm{A}]$.

Table 13. Resistive shunt and custom amplifier characterization performed for different values of the reference current supplied by the Fluke 6105A Calibrator.

\begin{tabular}{ccccc}
\hline $\begin{array}{c}K_{i} \\
{[/]}\end{array}$ & $\begin{array}{c}\sigma_{K i} \\
{[/]}\end{array}$ & $\begin{array}{c}\boldsymbol{\varphi}_{\boldsymbol{i}} \\
{[\mathbf{r a d}]}\end{array}$ & $\begin{array}{c}\sigma_{\varphi i} \\
{[/]}\end{array}$ & $\begin{array}{c}\boldsymbol{I}_{\text {cal }} \\
{[\mathbf{A}]}\end{array}$ \\
\hline 1.002674 & $4 \cdot 10^{-6}$ & $3 \cdot 10^{-6}$ & $2 \cdot 10^{-6}$ & 0.5 \\
1.002728 & $2 \cdot 10^{-6}$ & $-4.4 \cdot 10^{-5}$ & $3 \cdot 10^{-6}$ & 1 \\
1.002738 & $1 \cdot 10^{-6}$ & $-5.75 \cdot 10^{-5}$ & $6 \cdot 10^{-7}$ & 2 \\
1.002737 & $7 \cdot 10^{-6}$ & $-3.86 \cdot 10^{-5}$ & $9 \cdot 10^{-7}$ & 3 \\
1.002695 & $5 \cdot 10^{-6}$ & $-3.92 \cdot 10^{-5}$ & $7 \cdot 10^{-7}$ & 4 \\
1.002587 & $2 \cdot 10^{-6}$ & $-3.82 \cdot 10^{-5}$ & $7 \cdot 10^{-7}$ & 5 \\
1.002158 & $1 \cdot 10^{-6}$ & $-3.83 \cdot 10^{-5}$ & $7 \cdot 10^{-7}$ & 6 \\
\hline
\end{tabular}

In the same way, as stated for the voltage, these values obtained from the characterization of the current branch will be used to correct the measurements results obtained in Section 5.3 .

\subsection{SAMU Characterization Results}

In this last section of findings, the results obtained from the characterization of the SAMU are presented.

\subsubsection{Amplitude Characterization Tests Results}

These first tests were performed to evaluate the voltages and currents acquired by the SAMU.

A combination of $80 \%, 100 \%$, and $120 \%$ of the rated voltage $(57 \mathrm{~V})$, and $5 \%, 20 \%$, and $100 \%$ of the rated current $(5 \mathrm{~A})$, were applied from the reference Fluke $6105 \mathrm{~A}$ Calibrator.

The outcomes shown in Tables 14 and 15 take into account the results obtained from the characterization of the voltage divider, as well as the resistive shunt and the amplifier (previous Tables 12 and 13).

Table 14 reports, in the first column, the voltage applied from the calibrator $\left(V_{\text {cal }}\right)$; then, the measured voltage $\left(V_{m}\right)$ and its standard deviation of the mean $\left(\sigma_{m v}\right)$ are reported. Finally, the last two columns show the relative error in [\%] with respect to the reference source $\left(\varepsilon_{v}\right)$, as well as its standard deviation $\left(\sigma_{\varepsilon v}\right)$. The relative error was computed according to (1). 
Table 14. Results of the characterization of the SAMU applying voltages from the Fluke 6105A Calibrator.

\begin{tabular}{ccccc}
\hline$V_{\text {cal }}$ & $\begin{array}{c}\boldsymbol{V}_{\boldsymbol{m}} \\
{[\mathbf{V}]}\end{array}$ & $\begin{array}{c}\sigma_{m v} \\
{[\mathbf{V}]}\end{array}$ & $\begin{array}{c}\boldsymbol{\varepsilon}_{v} \\
{[\%]}\end{array}$ & $\begin{array}{c}\sigma_{\varepsilon v} \\
{[\%]}\end{array}$ \\
\hline 68$]$ & 68.37039 & $2 \cdot 10^{-5}$ & 0.04330 & $3 \cdot 10^{-5}$ \\
57 & 56.97651 & $2 \cdot 10^{-5}$ & 0.04121 & $3 \cdot 10^{-5}$ \\
45.6 & 45.58698 & $2 \cdot 10^{-5}$ & 0.02855 & $4 \cdot 10^{-5}$ \\
\hline
\end{tabular}

Table 15. Results of the characterization of the SAMU applying currents from the Fluke 6105A Calibrator.

\begin{tabular}{ccccc}
\hline $\begin{array}{c}\boldsymbol{I}_{\text {cal }} \\
{[\mathbf{A}]}\end{array}$ & $\begin{array}{c}\boldsymbol{I}_{\boldsymbol{m}} \\
{[\mathbf{A}]}\end{array}$ & $\begin{array}{c}\sigma_{m i} \\
{[\mathbf{A}]}\end{array}$ & $\begin{array}{c}\boldsymbol{\varepsilon}_{\boldsymbol{i}} \\
{[\%]}\end{array}$ & $\begin{array}{c}\sigma_{\varepsilon i} \\
{[\%]}\end{array}$ \\
\hline 5.0 & 4.99463 & $1 \cdot 10^{-5}$ & 0.10737 & $2 \cdot 10^{-5}$ \\
1.0 & 0.998875 & $5 \cdot 10^{-6}$ & 0.1125 & $5 \cdot 10^{-4}$ \\
0.250 & 0.249116 & $3 \cdot 10^{-6}$ & 0.354 & $1 \cdot 10^{-3}$ \\
\hline
\end{tabular}

In the same way, Table 15 shows, in the first column, the current applied from the calibrator $\left(I_{c a l}\right)$, and then the measured current $\left(I_{m}\right)$ and its standard deviation of the mean $\left(\sigma_{m i}\right)$ are reported. Finally, the last two columns show the relative error in [\%] with respect to the reference source $\left(\varepsilon_{i}\right)$, as well as its standard deviation $\left(\sigma_{\varepsilon i}\right)$. In this case, the relative error is calculated according to (1), but replacing the voltage with the current.

By comparing the results listed in Tables 14 and 15 with those in Table 8, it can be stated that the introduction of the resistive voltage divider does not decrease the accuracy of the measurement chain; hence, it confirms the SAMU voltage channel performance in terms of amplitude.

\subsubsection{Phase Characterization Tests Results}

This section presents the results of the phase displacement between two channels when both voltage and current are injected in the SAMU.

This test intends to evaluate the level of synchronism of the SAMU, taking into consideration the phase displacement introduced by voltage and current sensors (evaluated in 4.2 and whose results are presented in Section 5.2, Tables 12 and 13).

Table 16 presents, in the first column, the three voltages $(120 \%, 100 \%$ and $80 \%$ of the rated value) applied from the calibrator $\left(V_{\text {cal }}\right)$. In the second column, the three currents $\left(100 \%, 20 \%\right.$ and $5 \%$ of the rated value) are applied from the same reference source $\left(I_{\text {cal }}\right)$. The last two columns show the phase displacement in [rad] between the two channels $\left(\Delta_{\varphi}\right)$, computed as a mean over 500 measurements, and its standard deviation $\left(\sigma_{\Delta \varphi}\right)$.

Table 16. Results of the phase displacement between voltage and current channels of the SAMU, applying both signals from the Fluke 6105A Calibrator.

\begin{tabular}{cccc}
\hline $\begin{array}{c}V_{\text {cal }} \\
{[\mathrm{V}]}\end{array}$ & $\begin{array}{c}\boldsymbol{I}_{\text {cal }} \\
{[\mathbf{A}]}\end{array}$ & $\begin{array}{c}\Delta \varphi \\
{[\mathrm{rad}]}\end{array}$ & $\begin{array}{c}\sigma_{\Delta \varphi} \\
{[\mathrm{rad}]}\end{array}$ \\
\hline \multirow{3}{*}{68.4} & 5.0 & $2.20 \cdot 10^{-5}$ & $9 \cdot 10^{-7}$ \\
& 1.0 & $6.7 \cdot 10^{-5}$ & $3 \cdot 10^{-6}$ \\
& 0.250 & $1.1 \cdot 10^{-5}$ & $1 \cdot 10^{-5}$ \\
57 & 5.0 & $2.56 \cdot 10^{-5}$ & $9 \cdot 10^{-7}$ \\
& 1.0 & $7.4 \cdot 10^{-5}$ & $4 \cdot 10^{-6}$ \\
& 0.250 & $1.3 \cdot 10^{-4}$ & $1 \cdot 10^{-5}$ \\
45.6 & 5.0 & $1.86 \cdot 10^{-5}$ & $8 \cdot 10^{-7}$ \\
& 1.0 & $4.2 \cdot 10^{-5}$ & $4 \cdot 10^{-6}$ \\
& 0.250 & $1.7 \cdot 10^{-4}$ & $1 \cdot 10^{-5}$ \\
\hline
\end{tabular}

As demonstrated for amplitude, the high accuracy obtained from the characterization of the RTS itself (Table 9) is confirmed, even with the introduction of the current shunt and 
the amplifier. Of course, as has been previously noted, the phase displacement slightly worsens as the amplitude of the signal decreases.

It is worth highlighting here that the phase displacement of a single channel is the sum of two contributions: the RTS and the sensor. However, considering the negligible contribution of the sensors compared to the RTS, as described in Tables 12 and 13, the overall SAMU phase displacement can be considered to be the one in Table 9.

\subsubsection{Characterization Using Distorted Signals Results}

Considering the test described in Section 4.1.4 and its corresponding results presented in Table 11 in Section 5.1.4, this set of tests has been repeatedly applied to rated voltages and current to the SAMU.

With the same notation adopted in Table 11, Table 17 presents the voltage THD computed by the SAMU when a rated voltage with superimposed harmonics is applied to the SAMU by the reference calibrator.

Table 17. THD computed by the SAMU for distorted voltage measurements performed at a rated value of $57 \mathrm{~V}$.

\begin{tabular}{ccccccc}
\hline \multirow{2}{*}{ Signal } & $\begin{array}{c}h \\
{[/]}\end{array}$ & $\begin{array}{c}V_{h} \\
{[\%]}\end{array}$ & $\begin{array}{c}T H D_{v, c a l} \\
{[/]}\end{array}$ & $\begin{array}{c}T H D_{c, v} \\
{[/]}\end{array}$ & $\begin{array}{c}\sigma_{c, v} \\
{[/]}\end{array}$ & $\begin{array}{c}d T H D_{v} \\
{[\%]}\end{array}$ \\
\hline $\mathrm{A}$ & 3 & 5 & 5 & 5.00252 & $5 \cdot 10^{-5}$ & 0.0504 \\
$\mathrm{~B}$ & 3 & 5 & 7.8 & 7.83113 & $5 \cdot 10^{-5}$ & 0.3991 \\
& 5 & 6 & & & & \\
$\mathrm{C}$ & 3 & 5 & & & & \\
& 5 & 6 & 9.3 & 9.30945 & $5 \cdot 10^{-5}$ & 0.1016 \\
& 7 & 5 & & & & \\
$\mathrm{D}$ & 3 & 5 & & & & \\
& 5 & 6 & & & & \\
& 7 & 5 & 9.4 & 9.43097 & $5 \cdot 10^{-5}$ & 0.3295 \\
& 9 & 1.5 & & & & \\
\hline
\end{tabular}

Signal E, in Table 11, is now applied to the SAMU as a rated current of 5 A with superimposed harmonics. Table 18 presents the results obtained from 500 THD measurements, reporting $h$ as the harmonic order; $I_{h}$ is the relative amplitude in [\%] of the $50 \mathrm{~Hz}$ component; $T H D_{i, c a l}$ is the THD applied by the calibrator; and $T H D_{c, i}$ is the one computed by the RTS, together with its standard deviation, $\sigma_{c, i}$. Finally, $d T H D_{\mathrm{i}}$ is the relative error in $[\%]$ between the two previous values.

Table 18. THD computed by the SAMU for distorted current measurements performed at a rated value of $5 \mathrm{~A}$.

\begin{tabular}{ccccccc}
\hline \multirow{2}{*}{ Signal } & $h$ & $\begin{array}{c}\boldsymbol{I}_{\boldsymbol{h}} \\
{[\%]}\end{array}$ & $\begin{array}{c}T H D_{i, c a l} \\
{[/]}\end{array}$ & $\begin{array}{c}T H D_{c, i} \\
{[/]}\end{array}$ & $\begin{array}{c}\sigma_{c, i} \\
{[/]}\end{array}$ & $\begin{array}{c}d T H D_{i} \\
{[\%]}\end{array}$ \\
\hline \multirow{6}{*}{$\mathrm{E}$} & 2 & & & & \\
& 3 & 2 & & & & \\
& 5 & 10 & & & & \\
& 7 & 10 & & & & \\
& 11 & 10 & & & & \\
& 13 & 3 & 18.7 & 18.6798 & $4 \cdot 10^{-4}$ & 0.1080 \\
& 17 & 3 & & & & \\
& 23 & 3 & & & & \\
& 25 & 2 & & & & \\
\hline
\end{tabular}

Comparing Tables 17 and 18 with Table 11, it is possible to conclude that the obtained results are comparable with each other. Moreover, the influence of the voltage and current sensors to the SAMU does not decrease SAMU performances. In fact, the worsening in accuracy can be quantified, in the worst case, in a few parts per ten thousand. 
As a final comment on the amplitude and phase tests, the SAMU shows high accuracy performance, either in rated or distorted signal conditions.

\subsubsection{Power Characterization Tests Results}

Tables 19 and 20 report the results obtained from the power tests described in Section 4.3.

Table 19. Power measurements at rated voltage and currents using a Fluke 6105A Calibrator for a range of power factors $(P F)$, ranging between 1.0 to 0.1 at 0.1 steps.

\begin{tabular}{|c|c|c|c|c|c|c|c|}
\hline $\begin{array}{l}V_{c a l} \\
{[\mathrm{~V}]}\end{array}$ & $\begin{array}{l}I_{c a l} \\
{[\mathbf{A}]}\end{array}$ & $\begin{array}{l}P F \\
{[/]}\end{array}$ & $\begin{array}{l}P_{c a l} \\
{[W]}\end{array}$ & $\begin{array}{l}P_{m} \\
{[W]}\end{array}$ & $\begin{array}{c}\sigma_{p} \\
{[\mathbf{W}]}\end{array}$ & $\begin{array}{c}\mathcal{E}_{p} \\
{[\%]}\end{array}$ & $\begin{array}{l}\sigma_{\varepsilon p} \\
{[\%]}\end{array}$ \\
\hline \multirow{10}{*}{57} & \multirow{10}{*}{5} & 1.0 & 285 & 283.9594 & $2 \cdot 10^{-4}$ & 0.36511 & $8 \cdot 10^{-5}$ \\
\hline & & 0.9 & 256.5 & 255.5663 & $3 \cdot 10^{-4}$ & 0.3640 & $1 \cdot 10^{-4}$ \\
\hline & & 0.8 & 228 & 227.1918 & $3 \cdot 10^{-4}$ & 0.3545 & $1 \cdot 10^{-4}$ \\
\hline & & 0.7 & 199.5 & 198.8125 & $2 \cdot 10^{-4}$ & 0.3446 & $1 \cdot 10^{-4}$ \\
\hline & & 0.6 & 171 & 170.4221 & $2 \cdot 10^{-4}$ & 0.3380 & $1 \cdot 10^{-4}$ \\
\hline & & 0.5 & 142.5 & 141.9488 & $3 \cdot 10^{-4}$ & 0.3868 & $2 \cdot 10^{-4}$ \\
\hline & & 0.4 & 113.999 & 113.5872 & $3 \cdot 10^{-4}$ & 0.3621 & $3 \cdot 10^{-4}$ \\
\hline & & 0.3 & 85.502 & 85.2097 & $2 \cdot 10^{-4}$ & 0.3395 & $3 \cdot 10^{-4}$ \\
\hline & & 0.2 & 57 & 56.8092 & $2 \cdot 10^{-4}$ & 0.3347 & $4 \cdot 10^{-4}$ \\
\hline & & 0.1 & 28.499 & 28.4055 & $2 \cdot 10^{-4}$ & 0.3316 & $9 \cdot 10^{-4}$ \\
\hline
\end{tabular}

Table 20. Power measurements at $120 \%, 100 \%$ and $80 \%$ of the rated voltage, and at $100 \%, 20 \%$ and $5 \%$ of the rated current at unitary power factor $(\mathrm{PF})$.

\begin{tabular}{ccccccc}
\hline $\begin{array}{c}V_{\text {cal }} \\
{[\mathbf{V}]}\end{array}$ & $\begin{array}{c}\boldsymbol{I}_{\text {cal }} \\
{[\mathbf{A}]}\end{array}$ & $\begin{array}{c}\boldsymbol{P}_{\text {cal }} \\
{[\mathbf{W}]}\end{array}$ & $\begin{array}{c}\boldsymbol{P}_{m} \\
{[\mathbf{W}]}\end{array}$ & $\begin{array}{c}\sigma_{p} \\
{[\mathbf{W}]}\end{array}$ & $\begin{array}{c}\varepsilon_{p} \\
{[\%]}\end{array}$ & $\begin{array}{c}\sigma_{\varepsilon p} \\
{[\%]}\end{array}$ \\
\hline \multirow{3}{*}{68.4} & 5.0 & 342 & 340.6628 & $7 \cdot 10^{-4}$ & 0.3910 & $2 \cdot 10^{-4}$ \\
& 1.0 & 68.4 & 68.1076 & $4 \cdot 10^{-4}$ & 0.4275 & $5 \cdot 10^{-4}$ \\
& 0.250 & 17.1 & 16.9868 & $2 \cdot 10^{-4}$ & 0.662 & $1 \cdot 10^{-3}$ \\
57 & 5.0 & 285 & 283.9594 & $2 \cdot 10^{-4}$ & 0.36511 & $8 \cdot 10^{-5}$ \\
& 1.0 & 57 & 56.7538 & $3 \cdot 10^{-4}$ & 0.43199 & $6 \cdot 10^{-5}$ \\
& 0.250 & 14.25 & 14.1513 & $2 \cdot 10^{-4}$ & 0.692 & $1 \cdot 10^{-3}$ \\
45.6 & 5.0 & 228 & 227.0986 & $5 \cdot 10^{-4}$ & 0.3954 & $2 \cdot 10^{-4}$ \\
& 1.0 & 45.6 & 45.4130 & $3 \cdot 10^{-4}$ & 0.4102 & $6 \cdot 10^{-4}$ \\
& 0.250 & 11.4 & 11.3249 & $1 \cdot 10^{-4}$ & 0.659 & $1 \cdot 10^{-3}$ \\
\hline
\end{tabular}

Table 19 illustrates the voltage and current applied from the calibrator $\left(V_{\text {cal }}, I_{c a l}\right)$, the power factor $(P F)$, the power set in the calibrator $\left(P_{c a l}\right)$, the measured power $\left(P_{m}\right)$, and its standard deviation $\left(\sigma_{p}\right)$. In the last two columns, the relative error of the measured power $\left(\varepsilon_{p}\right)$, with respect to the one set in the calibrator, is presented, together with its standard deviation $\left(\sigma_{\varepsilon p}\right)$. For each value of PF, 500 measurements were performed.

The relative error is computed, as illustrated in (1), but, in this case, it is related to powers:

$$
\varepsilon_{p}=\frac{P_{m}-P_{c a l}}{P_{c a l}} \cdot 100,
$$

A first comment is that the power measurements, performed by the SAMU and quantified with $\varepsilon_{p}$, are very accurate for all the test values. Furthermore, as appreciable from $\varepsilon_{p}$, the influence of the PF on measured power is minimal. Therefore, the developed SAMU can be also used as a wattmeter (or even an energy meter).

Finally, Table 20 provides the results achieved from the power measurements at $120 \%$, $100 \%$ and $80 \%$ of the rated voltage (corresponding to $68.4,57$ and $45.6 \mathrm{~V}$, respectively) for a unitary power factor $(P F=1)$, and for a series of values of the current: $100 \%, 20 \%$ and $5 \%$ of the rated current, corresponding to 5, 1 and $0.250 \mathrm{~A}$, respectively.

The results in Table 20 are coherent with those described in Table 19. A further comment can be made on the behavior of $\varepsilon_{p}$ vs. the amplitude of the current and voltage 
signals. In fact, $\varepsilon_{p}$ increases when the current value decreases. This behavior, even if limited to few parts per thousand, is due to the fact that low voltage levels correspond to input voltages for the RTS, even when limited; hence, far from its full scale.

Overall, considering all the power measurements, the developed SAMU can be considered very accurate, even for the power measurements.

\subsection{Final Discussion}

This section aims at summarizing the findings at the end of the SAMU characterization. In particular, it has been proven that the developed SAMU has all the characteristics to be called such. Furthermore, its accuracy is high enough to be included in the $0.5 \mathrm{AC}$ (mainly due to phase displacement).

The characterization procedure confirmed the high performance of the processing unit adopted for the SAMU. In addition, such performance is not affected by the introduction of voltage and current sensors into the measurement chain. Regarding this aspect, the choice of very accurate sensors, made for the SAMU development, is not mandatory. In fact, less accurate sensors can be adopted (or whatever other available sensors are in the lab) because, after the characterization process, all systematic contributors to the uncertainty can be removed. However, by following the choice of very accurate voltage and current sensors, it is possible to neglect their effect on overall accuracy.

The SAMU has been tested with rated and distorted/real signals. From the results, it emerges that even the most distorted signals do not influence the measurement capabilities of the SAMU. This can be considered crucial in a power quality measurement environment; in fact, it can be excluded that, in real conditions, voltages and currents measured from the network are ideal as $50 \mathrm{~Hz}$ signals.

Finally, the developed SAMU has also been tested to assess its power measurement feature. The results clearly show that the accuracy obtained places the SAMU at the same level of the most accurate wattmeters on the market. It is worth highlighting that the SAMU does not have power and energy capabilities from a standard perspective. However, considering the several ancillary (or not) services used and implemented by DSOs, the information of the active and reactive power provides a significant added value to a particular device.

\section{Conclusions}

This work introduces a different idea of a SAMU. It consists of a real time simulator, which exploits the hardware-in-the-loop technology, plus voltage and current sensors. After a detailed description of all the SAMU components, the characterization procedure for the SAMU testing has been detailed and commented upon. The characterization aimed at testing the metrological performance of the SAMU in terms of amplitude, phase, and power, starting from sinusoidal or distorted/real signals. The results clearly confirm the high accuracy of the developed SAMU and its applicability in a typical laboratory environment. Therefore, this work succeeded in the obtaining of two main goals: (i) introducing a SAMU that features several measurement functions, even outside the SAMU requirements; and (ii) describing a simple and effective characterization procedure that can be replicated on every SAMU or synchronized measurement device.

Author Contributions: Conceptualization, A.M. and R.T.; methodology, L.P.; software, F.C.; validation, R.T.; formal analysis, A.M.; investigation, F.C.; resources, L.P.; data curation, F.C.; writing-original draft preparation, A.M.; writing-review and editing, F.C. All authors have read and agreed to the published version of the manuscript.

Funding: This research was funded by Future Grid II, grant number 17IND06. This project 17IND06 FutureGrid II received funding from the EMPIR programme, and was co-financed by the Participating States and the European Union's Horizon 2020 research and innovation programme.

Institutional Review Board Statement: Not applicable.

Informed Consent Statement: Not applicable. 


\section{Data Availability Statement: Not applicable.}

Conflicts of Interest: The authors declare no conflict of interest. The funders had no role in the design of the study; in the collection, analyses, or interpretation of data; in the writing of the manuscript, or in the decision to publish the results.

\section{References}

1. Power Distribution in EUROPE. Available online: https://cdn.eurelectric.org/media/1835/dso_report-web_final-2013-030-076 4-01-e-h-D66B0486.pdf (accessed on 22 February 2021).

2. Cheetham, P.; Hellany, A.; Jones, S. Density monitoring of high-voltage SF6 circuit breakers. IEEE Electr. Insul. Mag. 2015, 31, 6-13. [CrossRef]

3. Donner, G.; Smith, J.D.; Maldonado, J.J. An introduction to IEEE STD $1458^{\mathrm{TM}}-2017$ 'recommended practice for the selection, field testing, and life expectancy of molded-case circuit breakers for industrial applications'. In Proceedings of the Paper presented at the Record of Conference Papers-Annual Petroleum and Chemical Industry Conference, Cincinnati, OH, USA, 24-26 September 2018; pp. 47-52.

4. Zhao, S.; Yan, X.; Wang, B.; Wang, E.; Ma, L. Research on reliability evaluation method of DC circuit breaker based on markov model. Electr. Power Syst. Res. 2019, 173, 1-5. [CrossRef]

5. Al Marzooqi, A.; Packirisamy, N.; Hussin, M. Smart cable termination rectification program to enhance reliability of dubai electrical infrastructure. In Proceedings of the Smart Cities Symposium 2018, Manama, Bahrain, 22-23 April 2018. (CP747).

6. Gentilini, I.; Calone, R.; Giammanco, F.; Bolcato, G.; Weichold, J.; Stalder, M. The smart termination: An innovative component to enable smart grids development. In Proceedings of the 22nd International Conference and Exhibition on Electricity Distribution (CIRED 2013), Stockholm, Sweden, 10-13 June 2013. (615 CP).

7. Mingotti, A.; Ghaderi, A.; Peretto, L.; Tinarelli, R.; Lama, F. Test setup design, and calibration for tan delta measurements on MV cable joints. In Proceedings of the 9th IEEE International Workshop on Applied Measurements for Power Systems (AMPS), Bologna, Italy, 26-28 September 2018.

8. Peretto, L.; Tinarelli, R.; Ghaderi, A.; Mingotti, A.; Mazzanti, G.; Valtorta, G.; Danesi, S. Monitoring cable current and laying environment parameters for assessing the aging rate of MV cable joint insulation. In Proceedings of the 2018 IEEE Conference on Electrical Insulation and Dielectric Phenomena (CEIDP), Cancun, Mexico, 21-24 October 2018; pp. $390-393$.

9. Kalantari Khandani, M.; Askarzadeh, A. Optimal MV/LV transformer allocation in distribution network for power losses reduction and cost minimization: A new multi-objective framework. Int. Trans. Electr. Energy Syst. 2020, 30, e12361. [CrossRef]

10. Lefort, R.; Taquet, B.; Vauzelle, R.; Courtecuisse, V.; Poussard, A.M.; Idir, N. High frequency MV/LV transformer modelling for power line communication applications. In Proceedings of the 18th IEEE International Symposium on Power Line Communications and Its Applications, Glasgow, UK, 30 March-2 April 2014; pp. 30-35.

11. Nikolić, B.; Khan, S. Modelling and optimisation of design of non-conventional instrument transformers. J. Phys. Conf. Ser. 2019, 1379, 012057. [CrossRef]

12. Torre, F.D.; Faifer, M.; Morando, A.P.; Ottoboni, R.; Cherbaucich, C.; Gentili, M.; Mazza, P. Instrument transformers: A different approach to their modeling. In Proceedings of the 2011 IEEE International Workshop on Applied Measurements for Power Systems (AMPS), Aachen, Germany, 28-30 September 2011; pp. 37-41.

13. Mingotti, A.; Peretto, L.; Tinarelli, R. A simple modelling procedure of rogowski coil for power systems applications. In Proceedings of the 2019 10th IEEE International Workshop on Applied Measurements for Power Systems (AMPS), Aachen, Germany, 25-27 September 2019.

14. Chen, Y.; Mohns, E.; Badura, H.; Rather, P.; Luiso, M. Setup and characterisation of reference current-to-voltage transformers for wideband current transformers calibration up to $2 \mathrm{kA}$. In Proceedings of the 2019 10th IEEE International Workshop on Applied Measurements for Power Systems (AMPS), Aachen, Germany, 25-27 September 2019.

15. Letizia, P.S.; Crotti, G.; Giordano, D.; Delle Femine, A.; Gallo, D.; Landi, C.; Luiso, M. Low cost procedure for frequency characterization of voltage instrument transformers. In Proceedings of the 2019 IEEE International Instrumentation and Measurement Technology Conference (I2MTC), Auckland, New Zealand, 20-23 May 2019.

16. Faifer, M.; Laurano, C.; Ottoboni, R.; Toscani, S.; Zanoni, M. Characterization of voltage instrument transformers under nonsinusoidal conditions based on the best linear approximation. IEEE Trans. Instrum. Meas. 2018, 67, 2392-2400. [CrossRef]

17. Mingotti, A.; Peretto, L.; Tinarelli, R.; Zhang, J. Use of COMTRADE fault current data to test inductive current transformers. In Proceedings of the 2019 IEEE International Workshop on Metrology for Industry 4.0 and IoT (MetroInd 4.0 and IoT), Naples, Italy, 4-6 June 2019; pp. 103-107.

18. Cheng, S.; Zhang, G.; Guo, Z.; Shen, Y.; Yu, W. Influence mechanism of conductor eccentricity on all-fiber optic current transformers. Dianli Xitong Zidonghua/Autom. Electr. Power Syst. 2015, 39, 137-143.

19. Draxler, K.; Ulvr, M.; Styblikova, R.; Hlavacek, J. Calibration of burdens for instrument transformers. In Proceedings of the International Instrumentation and Measurement Technology Conference (I2MTC), Dubrovnik, Croatia, 25-28 May 2020.

20. Misak, S.; Fulnecek, J. The influence of ferroresonance on a temperature of voltage transformers in undeground mines. In Proceedings of the 2017 18th International Scientific Conference on Electric Power Engineering (EPE), Kouty nad Desnou, Czech Republic, 17-19 May 2017. 
21. Wu, W.; Xu, Y.; Yang, S.; Lu, S.; Xiao, X. Research on proximity magnetic field influence in measuring error of active electronic current transformers. In MATEC Web of Conferences; EDP Sciences: Ulis, France, 2016; Volume 59, p. 01010.

22. Mingotti, A.; Peretto, L.; Tinarelli, R.; Ghaderi, A. Uncertainty sources analysis of a calibration system for the accuracy vs. temperature verification of voltage transformers. J. Phys. Conf. Ser. 2018, 1065, 052041. [CrossRef]

23. IEC 61869-1. Part 1: General requirements. In Instrument Transformers; International Standardization Organization: Geneva, Switzerland, 2010.

24. IEC 61869-6. Part 6: Additional general requirements for low-power instrument transformers. In Instrument Transformers; International Standardization Organization: Geneva, Switzerland, 2016.

25. IEC 61869-2. Part 2: Additional requirements for current transformers. In Instrument Transformers; International Standardization Organization: Geneva, Switzerland, 2012.

26. IEC 61869-3. Part 3: Additional requirements for inductive voltage transformers. In Instrument Transformers; International Standardization Organization: Geneva, Switzerland, 2011.

27. IEC 61869-10. Part 10: Additional requirements for low-power passive current transformers. In Instrument Transformers; International Standardization Organization: Geneva, Switzerland, 2018.

28. IEC 61869-11. Part 11: Additional requirements for low-power passive voltage transformers. In Instrument Transformers; International Standardization Organization: Geneva, Switzerland, 2018.

29. Cipolletta, G.; Delle Femine, A.; Gallo, D.; Landi, C.; Luiso, M. Design approach for a stand alone merging unit. In Proceedings of the 16th IMEKO TC10 Conference on Testing, Diagnostics \& Inspection as a comprehensive value chain for Quality \& Safety, Berlin, Germany, 3-4 September 2019; pp. 107-112.

30. Wang, Y.; Tan, J. Design of a synthesized merging unit based on IEC 61850-9-2. In Applied Mechanics and Materials; Trans Tech Publications Ltd.: Bäch SZ, Switzerland, 2013; Volume 241, pp. 2223-2227.

31. Han, Y.; Mu, L.; Zhuang, W. Design and application of new merging unit based on dual CPU. In Advanced Materials Research; Trans Tech Publications Ltd.: Bäch SZ, Switzerland, 2013; Volume 614, pp. 1724-1728. [CrossRef]

32. Liu, J.; Li, K.; Yang, H. The design of a merging unit of electronic transformer based on ARM. In Proceedings of the Universities Power Engineering Conference, Brighton, UK, 4-6 September 2007; pp. 712-716.

33. Cipolletta, G.; Femine, A.D.; Gallo, D.; Landi, C.; Luiso, M. Design and characterisation of a stand-alone merging unit. Acta IMEKO 2020, 9, 40-48. [CrossRef]

34. Strnad, I.; Pregrad, G.; Višić, I.; Gazivoda, S. Development and implementation of the smart measuring unit with MU and PMU functionalities for smart grid applications. In Proceedings of the 2018 1st International Colloquium on Smart Grid Metrology (SmaGriMet), Split, Croatia, 24-27 April 2018; pp. 1-5.

35. Agustoni, M.; Mortara, A. A calibration setup for iec 61850-9-2 devices. IEEE Trans. Instrum. Meas. 2017, 66, 1124-1130. [CrossRef]

36. Shu, Z.; Cai, X.; Chen, B.; Zhou, N.; Su, Y. Research of intelligent substation merging unit calibration equipment. In Proceedings of the IOP Conference Series: Materials Science and Engineering, Shanghai, China, 24-26 March 2017; Volume 199.

37. Cheng, H.M.; Huang, Q.F.; Ji, F.; Xu, Q.; Liu, J.; Tian, Z.Q. System for calibrating analogue merging units in absence of synchronization signals. Metrol. Meas. Syst. 2018, 25, 129-138.

38. Braun, J.P. Measure of the absolute phase angle of a power frequency sinewave with respect to UTC. In Proceedings of the Conference on Precision Electromagnetic Measurements (CPEM 2018), Paris, France, 8-13 July 2018.

39. Gurbiel, M.; Komarnicki, P.; Styczynski, Z.A.; Gatzen, F.W.; Dzienis, C. Merging unit accuracy testing. In Proceedings of the 2009 IEEE Power and Energy Society General Meeting, Calgary, AB, Canada, 26-30 July 2009.

40. Liu, H.T.; Huang, M.Y.; Zhang, Y.H. Merging unit performance evaluation of intelligent substation based on the triangular fuzzy number. In Advanced Materials Research; Trans Tech Publications Ltd.: Bäch SZ, Switzerland, 2014; Volume 989, pp. 5559-5564. [CrossRef]

41. IEC 61850-2. Part 2: Glossary. In Communication Networks and Systems for Power Utility Automation; International Standardization Organization: Geneva, Switzerland, 2019.

42. IEC 61869-13. Part 13: Stand-alone merging unit (SAMU). In Instrument Transformers; International Standardization Organization: Geneva, Switzerland, 2021.

43. IEC 61850-9-2. Part 9-2: Specific communication service mapping (SCSM)—Sampled values over ISO/IEC 8802-3. In Communication Networks and Systems for Power Utility Automation; International Standardization Organization: Geneva, Switzerland, 2020.

44. IEC 61869-9. Part 9: Digital interface for instrument transformers. In Instrument Transformers; International Standardization Organization: Geneva, Switzerland, 2019.

45. Liu, D.; Xu, L.; Liu, P. Strategy and mechanism of time and sampling synchronization of digital substation in China. In Proceedings of the 2019 3rd IEEE Conference on Energy Internet and Energy System Integration: Ubiquitous Energy Network Connecting Everything (EI2), Changsha, China, 8-10 November 2019; pp. 1816-1821.

46. Roux, L.D.; Qastalane, Z.; Leitloff, V. Tests to assess 61850-9-2 based stand alone merging units for metering purposes. In Proceedings of the 3th International Conference on Development in Power System Protection 2016 (DPSP), 7-10 March 2016. (CP671).

47. 50160 BS EN 50160:2010+A1. Voltage Characteristics of Electricity Supplied by Public Electricity Networks; BSI Standards Publication: London, UK, 2015. 